\title{
One-Sided Extendability and $p$-Continuous Analytic Capacities
}

\author{
E. Bolkas ${ }^{1,3}$ - V. Nestoridis ${ }^{1}$ - C. Panagiotis ${ }^{1,4}$. \\ M. Papadimitrakis ${ }^{2}$
}

Received: 16 April 2018 / Published online: 6 June 2018

(C) The Author(s) 2018

\begin{abstract}
Using complex methods combined with Baire's Theorem, we show that onesided extendability, extendability, and real analyticity are rare phenomena on various spaces of functions in the topological sense. These considerations led us to introduce the $p$-continuous analytic capacity and variants of it, $p \in\{0,1,2, \ldots\} \cup\{\infty\}$, for compact or closed sets in $\mathbb{C}$. We use these capacities in order to characterize the removability of singularities of functions in the spaces $A^{p}$.
\end{abstract}

Keywords Locally injective curve $\cdot$ Extendability $\cdot$ Real analyticity $\cdot$ Continuous analytic capacity · Montel's Theorem · Baire's Theorem

Dedicated to the memory of Professor Jean-Pierre Kahane.

V. Nestoridis

vnestor@math.uoa.gr

E. Bolkas

lefterisbolkas@gmail.com; ebolkas@math.princeton.edu

C. Panagiotis

chris_panagiwtis@hotmail.gr; C.Panagiotis@warwick.ac.uk

M. Papadimitrakis

papadim@math.uoc.gr

1 Department of Mathematics, University of Athens, 15784 Panepistemiopolis, Athens, Greece

2 Department of Mathematics and Applied Mathematics, University of Crete, Voutes Campus, 70013 Heraklion, Crete, Greece

3 Present Address: Department of Mathematics, Princeton University, Fine Hall, Washington Road, Princeton, NJ 08544-1000, USA

4 Mathematics Institute, University of Warwick, Coventry CV4 7AL, UK 
Mathematics Subject Classification $30 \mathrm{H05} \cdot$ 53A04 $\cdot$ 30C85

\section{Introduction}

In [3] it is proven that the set $X$ of nowhere analytic functions in $C^{\infty}([0,1])$ contains a dense and $G_{\delta}$ subset of $C^{\infty}([0,1])$. In [2] using Fourier methods it is shown that $X$ is itself a dense and $G_{\delta}$ subset of $C^{\infty}([0,1])$. Furthermore, combining the above methods with Borel's Theorem [8] and a version of Michael's Selection Theorem [12] the above result has been extended to $C^{\infty}(\gamma)$, where $\gamma$ is any analytic curve. In the case where $\gamma$ is the unit circle $T$ every function $f \in C^{\infty}(T)$ can be written as a sum $f=g+w$ where $g$ belongs to $A^{\infty}(D)$ and is holomorphic on the open unit disk $D$ and very smooth up to the boundary and $w$ has similar properties in $D^{c}$. Now if we assume that $f$ is extendable somewhere towards one side of $T$, say in $D^{c}$, then because $w$ is regular there, it follows that $g \in A^{\infty}(D)$ is extendable. But the phenomenon of somewhere extendability has been proven to be a rare phenomenon in the Fréchet space $A^{\infty}(D)$ [9]. It follows that the phenomenon of one-sided somewhere extendability is a rare phenomenon in $C^{\infty}(T)$ or more generally in $C^{p}(\gamma), p \in\{\infty\} \cup\{0,1,2, \ldots\}$ for any analytic curve $\gamma[2]$.

After the preprint [2] has been circulated, P. Gautier noticed that the previous result holds more generally for Jordan arcs without the assumption of analyticity of the curve. Indeed, applying complex methods appearing in the last section of [2] we prove this result. It suffices to use the Oswood-Caratheodory Theorem combined with Montel's Theorem and the Poisson integral formula applied to the boundary values of bounded holomorphic functions in $H^{\infty}(D)$. In fact this complex method is most natural to our considerations of extendability, real analyticity, and one-sided extendability. The proofs are simplified, and the results hold under much more general assumptions than the assumptions imposed by the Fourier method. This complex method is developed in the present paper.

In Sect. 4, we prove that extendability and real analyticity are rare phenomena in various spaces of functions on locally injective curves $\gamma$. For the real analyticity result, we assume that $\gamma$ is analytic and the result holds in any $C^{k}(\gamma), k \in\{0,1,2,3, \ldots\} \cup$ $\{\infty\}$ endowed with its nature topology. For the other results, the phenomena are proven to be rare in $C^{k}(\gamma)$ provided that the locally injective curve $\gamma$ has smoothness at least of degree $k$.

In Sect. 5, initially, we consider a finite set of disjoint curves $\gamma_{1}, \gamma_{2}, \ldots, \gamma_{n}$. Then in the case where $\gamma_{1}, \gamma_{2}, \ldots, \gamma_{n}$ are disjoint Jordan curves in $\mathbb{C}$ bounding a domain $\Omega$ of finite connectivity, we consider the spaces $A^{p}(\Omega), p \in\{0,1,2,3, \ldots\} \cup\{\infty\}$ which by the maximum principle can be seen as function spaces on $\partial \Omega=\gamma_{1}^{*} \cup \cdots \cup \gamma_{n}^{*}$. In these spaces we show that the above phenomena of extendability or real analyticity are rare. For the real analyticity result, we assume analyticity of $\partial \Omega$, but for the extendability result we do not need to assume any smoothness of the boundary.

In Sect. 6, we consider the one-sided extendability from a locally injective curve $\gamma$ and we prove that this is a rare phenomenon in various spaces of functions. We construct a denumerable family $G_{n}$ of Jordan domains $G$ containing in their boundary a non-trivial arc $J$ of the image $\gamma^{*}$ of $\gamma$, such that each other domain $\Omega$ with similar 
properties contains some $G_{n}$. We show that the phenomenon of extendability is rare for each domain $G$. Then by denumerable union (or intersection of the complements), we obtain our result with the aid of Baire's Category Theorem. We mention that the one-sided extendability of a function $f: \gamma^{*} \rightarrow \mathbb{C}$ is meant as the existence of a function $F: G \cup J \rightarrow \mathbb{C}$ which is holomorphic on the Jordan domain $G$, continuous on $G \cup J$ and such that on the arc $J$ of $\gamma^{*}$ we have $\left.F\right|_{J}=\left.f\right|_{J}$. Such notions of one-sided extendability have been considered in [4] and the references therein, but in the present article and [2] it is, as far as we know, the first time where the phenomenon is proven to be rare.

At the end of Sect. 6, we prove similar results on one-sided extendability on the space $A^{p}(\Omega)$, where $\Omega$ is a finitely connected domain in $\mathbb{C}$ bounded by a finite set of disjoint Jordan curves $\gamma_{1}, \gamma_{2}, \ldots, \gamma_{n}$. Now the extension $F$ of a function $f \in A^{p}(\Omega)$ has to coincide with $f$ only on a non-trivial arc of the boundary of $\Omega$, not on an open subset of $\Omega$. Certainly if the continuous analytic capacity of $\partial \Omega$ is zero, the latter automatically happens, but not in general.

In Sect. 7, we consider a domain $\Omega$ in $\mathbb{C}$, a compact subset $L$ of $\Omega$, and we study the phenomenon of extendability of a function $f \in A^{p}(\Omega \backslash L)$ to a function $F$ in $A^{p}(\Omega)$. There is a dichotomy. Either for every $f$ this is possible or generically for all $f \in A^{p}(\Omega \backslash L)$ this fails. In order to characterize when each horn of the above dichotomy holds we are led to define the $p$-continuous analytic capacity $a_{p}(L)$ $(p \in\{0,1,2,3, \ldots\} \cup\{\infty\})$, where $a_{0}(L)$ is the known continuous analytic capacity $a_{0}(L)=a(L)$ [7].

The study of the above capacities and variants of it is done in Sect. 3. For $p=1$, the $p$-continuous analytic capacity $a_{1}$ is distinct from the continuous analytic capacity $a_{0}=a$. In particular if $K_{1 / 3}$ is the usual Cantor set lying on $[0,1]$ and $L=K_{1 / 3} \times K_{1 / 3}$, then $a_{0}(L)>0$, but $a_{1}(L)=0$. This means that for any open set $U$ containing $L$ there exists a function in $A(U \backslash L)$ which is not holomorphic on $U$, but if the derivative of a function in $A(U \backslash L)$ extends continuously on $L$, then the function is holomorphic on $U$. Generic versions of this fact imply that $A^{1}(U \backslash L)$ is of first category in $A^{0}(U \backslash L)=A(U \backslash L)$. If we replace the spaces $A^{p}$ with the $\tilde{A}^{p}$ spaces of Whitney type, then the extension on $L$ is equivalent to the fact that the interior of $L$ is void. Thus we can define the continuous analytic capacities $\tilde{a}^{p}(L)$ which vanish if and only if the interior of $L$ is empty. We prove what is needed in Sect. 7. More detailed study of those capacities will be done in future papers; for instance, we can investigate the semiadditivity of $a_{p}$, whether the vanishing of $a_{p}$ on a compact set $L$ is a local phenomenon and whether replacing the continuous analytic capacity $a$ by the Ahlfors analytic capacity $\gamma$ we can define capacities $\gamma_{p}$ satisfying the analogous properties. Certainly the spaces $A^{p}(\Omega)$ will be replaced by $H_{p}^{\infty}(\Omega)$, the space of holomorphic functions on $\Omega$ such that for every $l \in \mathbb{N}, l \leq p$ the derivative $f^{(l)}$ of order $l$ is bounded on $\Omega$. We will also examine if a dichotomy result as in Sect. 7 holds for the spaces $H_{p}^{\infty}(\Omega)$ in the place of $A^{p}(\Omega)$. All these in future papers.

In Sect. 2, some preliminary geometry of locally injective curves is presented; for instance, a curve is real analytic if and only if real analyticity of a function on the curve is equivalent to holomorphic extendability of the function on disks centered on points of the curve. We also show that if the map $\gamma$ defining the curve is a homeomorphism 
with non-vanishing derivative, then the spaces $C^{k}(\gamma), k \in\{0,1,2,3, \ldots\} \cup\{\infty\}$ are independent of the particular parametrization $\gamma$ and depend only on the image $\gamma^{*}$ of $\gamma$. Thus in some cases it makes sense to write $C^{k}(\partial \Omega)$ and prove generic results in these spaces.

Finally, we mention that some of the results of Sect. 5 are valid for analytic curves $\gamma$; that is, they hold when we use a conformal parametrization of $\gamma$. Naturally comes the question whether these results remain true if we change the parametrization of the curve; in particular what happens if we consider the parametrization with respect to the arc length $s$ ? Answering this question was the motivation of [10] where it is proven that arc length is a global conformal parameter for any analytic curve. Thus the results of Sect. 5 remain true if we use the arc length parametrization. Finally, we mention that in the present paper we start with qualitative categorical results, which lead us to quantitative notions as the $p$-continuous analytic capacity $a_{p}$ and the $p$-analytic capacity $\gamma_{p}$. A preliminary version of this paper can be found in [1], where some proofs are more detailed than in the present paper.

\section{Preliminaries}

In most of our results it is important what is the degree of smoothness of a curve and the relation of real analyticity of functions on a curve with the holomorphic extendability of them around the curve. That is why we present here some basic results concerning locally injective curves in $\mathbb{C}$.

Unless otherwise specified $I$ is an interval and $X$ is an interval or the unit circle.

Definition 2.1 Let $\gamma: X \rightarrow \mathbb{C}$ be a continuous and locally injective function and $l \in$ $\{1,2,3, \ldots\} \cup\{\infty\}$. The curve $\gamma$ belongs to the class $C^{l}(X)$, if for $k \in\{1,2,3, \ldots\}, k \leq$ $l$, the derivative $\gamma^{(k)}$ exists, and is a continuous function.

Definition 2.2 Let $\gamma: X \rightarrow \mathbb{C}$ be a continuous and locally injective function and $k \in\{1,2,3, \ldots\} \cup\{\infty\}$. A function $f: \gamma^{*} \rightarrow \mathbb{C}$ defined on the image $\gamma^{*}=\gamma(X)$ belongs to the class $C^{k}(\gamma)$ if for every $l \in\{1,2,3, \ldots\},, l \leq k$ the derivative $(f \circ \gamma)^{(l)}$ exists, and is a continuous function. Let $\left(X_{n}\right), n \in\{1,2,3, \ldots\}$ be an increasing sequence of compact intervals such that $\bigcup_{n=1}^{\infty} X_{n}=X$. The topology of the space $C^{k}(\gamma)$ is defined by the seminorms

$$
\sup _{t \in X_{n}}\left|(f \circ \gamma)^{(l)}(t)\right|, l=0,1,2, \ldots, k, n=1,2,3, \ldots
$$

In this way $C^{k}(\gamma)$ becomes a Banach space if $k<+\infty$ and $X$ is compact. Otherwise it is a Fréchet space. In every case, Baire's Theorem is at our disposal.

Definition 2.3 Let $\gamma: I \rightarrow \mathbb{C}$ be a continuous and locally injective function. We will say that the curve $\gamma$ is analytic at $t_{0} \in I$ if there exist an open set $t_{0} \in V \subseteq \mathbb{C}$, a real number $\delta>0$ with $\left(t_{0}-\delta, t_{0}+\delta\right) \cap I \subset V$, and a holomorphic and injective function $F: V \rightarrow \mathbb{C}$ such that $\left.F\right|_{\left(t_{0}-\delta, t_{0}+\delta\right)}=\left.\gamma\right|_{\left(t_{0}-\delta, t_{0}+\delta\right)}$. If $\gamma$ is analytic at every $t \in I$, we will say that $\gamma$ is an analytic curve. 
Lemma 2.4 Let $t_{0} \in I$ and $\gamma: I \rightarrow \mathbb{C}$ be a continuous and locally injective function. We suppose that for every function $f: I \rightarrow \mathbb{C}$ items $(1)$ and (2) are equivalent:

1. There exists a power series of a real variable $\sum_{n=0}^{\infty} a_{n}\left(t-t_{0}\right)^{n}, a_{n} \in \mathbb{C}$ with positive radius of convergence $r>0$ and $\delta \in(0, r]$, which coincides with $f$ on $\left(t_{0}-\right.$ $\left.\delta, t_{0}+\delta\right) \cap I$.

2. There exists a power series of a complex variable $G(z)=\sum_{n=0}^{\infty} b_{n}\left(z-\gamma\left(t_{0}\right)\right)^{n}$, $b_{n} \in \mathbb{C}$ with positive radius of convergence $s>0$ and $\epsilon \in(0, s]$ such that $f(t)=G(\gamma(t))$ for $t \in\left(t_{0}-\epsilon, t_{0}+\epsilon\right) \cap I$.

Then $\gamma$ is analytic at $t_{0}$.

Proof Implication (2) $\Rightarrow$ (1) will only be used to prove that $\gamma$ is differentiable on an open interval that contains $t_{0}$. We start by considering $\beta>0$ and $J=\left(t_{0}-\beta, t_{0}+\beta\right) \cap I$. For every $t \in J$ we choose $f(t)=\gamma(t)=\gamma\left(t_{0}\right)+\left(\gamma(t)-\gamma\left(t_{0}\right)\right)$ and so by $(2) \Rightarrow$ (1) we obtain that there exists $0<\delta<\beta$ such that

$$
\gamma(t)=\sum_{n=0}^{\infty} a_{n}\left(t-t_{0}\right)^{n}
$$

for some constants $a_{n} \in \mathbb{C}$ and for every $t \in\left(t_{0}-\delta, t_{0}+\delta\right) \cap I$. Therefore $\gamma$ is differentiable on this interval. Now for $g(t)=t=t_{0}+\left(t-t_{0}\right)$ by $(1) \Rightarrow(2)$ we have that there is $0<\epsilon \leq \delta$ such that

$$
t=\sum_{n=0}^{\infty} b_{n}\left(\gamma(t)-\gamma\left(t_{0}\right)\right)^{n}
$$

for some constants $b_{n} \in \mathbb{C}$ for every $t \in\left(t_{0}-\epsilon, t_{0}+\epsilon\right)$. Differentiation of the above equation at $t=t_{0}$ yields the relation $1=b_{1} \gamma^{\prime}\left(t_{0}\right)$ which implies that $b_{1} \neq 0$. The power series $\sum_{n=0}^{\infty} b_{n}\left(z-\gamma\left(t_{0}\right)\right)^{n}$ has a positive radius of convergence and so there exists $\alpha>0$ such that $\gamma(t) \in D\left(\gamma\left(t_{0}\right), \alpha\right)$ for every $t \in\left(t_{0}-\epsilon, t_{0}+\epsilon\right) \cap I$ and the function $f: D\left(\gamma\left(t_{0}\right), \alpha\right) \rightarrow \mathbb{C}$ with

$$
f(z)=\sum_{n=0}^{\infty} b_{n}\left(z-\gamma\left(t_{0}\right)\right)^{n}
$$

is holomorphic. Also, $f(\gamma(t))=t$ for every $t \in\left(t_{0}-\epsilon, t_{0}+\epsilon\right) \cap I$. Since $f^{\prime}\left(\gamma\left(t_{0}\right)\right)=$ $b_{1} \neq 0, f$ is locally invertible and thus the inverse, $h$, of $f$ is well defined on the open disk $D\left(t_{0}, \eta\right)$ where $0<\eta<\epsilon$. Moreover, $\gamma(t)=h(t)$ for every $t \in\left(t_{0}-\eta, t_{0}+\eta\right) \cap I$ and $h$ is holomorphic and injective.

Remark 2.5 The above proof shows that if $\gamma$ in Lemma 2.4 belongs to $C^{1}(I)$, then the conclusion of the lemma is true even if we only assume that $(1) \Rightarrow(2)$ is true. 
The following lemma is the inverse of Lemma 2.4.

Lemma 2.6 Let $t_{0} \in I$ and $\gamma: I \rightarrow \mathbb{C}$ be a continuous and locally injective function, which is analytic at $t_{0}$, and $f: I \rightarrow \mathbb{C}$. Then the following are equivalent:

1. There exists a power series of a real variable $\sum_{n=0}^{\infty} a_{n}\left(t-t_{0}\right)^{n}, a_{n} \in \mathbb{C}$ with positive radius of convergence $r>0$ and $\delta \in(0, r]$ which coincides with $f$ on $\left(t_{0}-\delta, t_{0}+\right.$ $\delta) \cap I$.

2. There exists a power series of a complex variable $G(z)=\sum_{n=0}^{\infty} b_{n}\left(z-\gamma\left(t_{0}\right)\right)^{n}, b_{n} \in$ $\mathbb{C}$ with positive radius of convergence $s>0$ and $\epsilon>0$ such that $f(t)=G(\gamma(t))$ for every $t \in\left(t_{0}-\epsilon, t_{0}+\epsilon\right) \cap I$.

Proof Let us start with the following observation. Since $\gamma$ is an analytic curve at $t_{0}$ there is an open disk $D\left(t_{0}, \varepsilon\right) \subseteq \mathbb{C}$, where $\varepsilon>0$ and a holomorphic and injective function $\Gamma: D\left(t_{0}, \varepsilon\right) \rightarrow \mathbb{C}$ with $\Gamma(t)=\gamma(t)$ for $t \in\left(t_{0}-\varepsilon, t_{0}+\varepsilon\right) \cap I$.

$(1) \Rightarrow(2)$ We define the function $g(z)=\sum_{n=0}^{\infty} a_{n}\left(z-t_{0}\right)^{n}, z \in D\left(t_{0}, \delta\right)$, which is holomorphic on $D\left(t_{0}, \delta\right)$. As $\Gamma^{-1}$ is holomorphic on $\Gamma\left(D\left(t_{0}, \varepsilon\right)\right)$, we are led to consider the function $F=g \circ \Gamma^{-1}$ and notice that $(F \circ \Gamma)(t)=f(t), t \in D\left(t_{0}, \varepsilon\right) \cap I$. It follows that there are $b_{n} \in \mathbb{C}, n \in \mathbb{N}$, and $\delta>0$ such that $F(z)=\sum_{n=0}^{\infty} b_{n}\left(z-\gamma\left(t_{0}\right)\right)^{n}$ for every $z \in D\left(\gamma\left(t_{0}\right), \delta\right) \subseteq \Gamma\left(D\left(t_{0}, \varepsilon\right)\right)$. Thus $f(t)=(F \circ \gamma)(t)=\sum_{n=0}^{\infty} b_{n}\left(\gamma(t)-\gamma\left(t_{0}\right)\right)^{n}$ in an interval $\left(t_{0}-s, t_{0}+s\right) \cap I$ where $s>0$.

$(2) \Rightarrow(1)$ We consider the function $G(z)=\sum_{n=0}^{\infty} b_{n}\left(z-\gamma\left(t_{0}\right)\right)^{n}, z \in D\left(\gamma\left(t_{0}\right), s\right)$. Choose $a>0$ with $a<\varepsilon$ such that $\Gamma\left(D\left(t_{0}, a\right)\right) \subseteq D\left(\gamma\left(t_{0}\right), s\right)$. The function $G \circ \Gamma$ : $D\left(t_{0}, a\right) \rightarrow \mathbb{C}$ is holomorphic. Therefore, there are $a_{n} \in \mathbb{C}, n \in \mathbb{N}$ such that

$$
(G \circ \Gamma)(z)=\sum_{n=0}^{\infty} a_{n}\left(z-t_{0}\right)^{n}, z \in D\left(t_{0}, a\right)
$$

and consequently

$$
f(t)=G(\gamma(t))=\sum_{n=0}^{\infty} a_{n}\left(t-t_{0}\right)^{n}, t \in\left(t_{0}-a, t_{0}+a\right) .
$$

Definition 2.7 Let $\gamma: I \rightarrow \mathbb{C}$ be a locally injective curve and $z_{0}=\gamma\left(t_{0}\right), t_{0} \in I$. A function $f: \gamma^{*} \rightarrow \mathbb{C}$ belongs to the class of non-holomorphically extendable at $\left(t_{0}, z_{0}=\gamma\left(t_{0}\right)\right)$ functions if there are no open disk $D\left(z_{0}, r\right), r>0$, and $\eta>0$ and a holomorphic function $F: D\left(z_{0}, r\right) \rightarrow \mathbb{C}$, such that $\gamma\left(\left(t_{0}-\eta, t_{0}+\eta\right) \cap I\right) \subset D\left(z_{0}, r\right)$ and $F(\gamma(t))=f(\gamma(t))$ for all $t \in\left(t_{0}-\eta, t_{0}+\eta\right) \cap I$. Otherwise, $f$ is holomorphically extendable at $\left(t_{0}, z_{0}=\gamma\left(t_{0}\right)\right)$. 
Definition 2.8 Let $\gamma: I \rightarrow \mathbb{C}$ be a continuous map and $t_{0} \in I$. A function $f: \gamma^{*} \rightarrow$ $\mathbb{C}$ is real analytic at $\left(t_{0}, z_{0}=\gamma\left(t_{0}\right)\right)$ if there exist $\delta>0$ and a power series $\sum_{n=0}^{\infty} a_{n}(t-$ $\left.t_{0}\right)^{n}$ with a radius of convergence $\epsilon>\delta>0$, such that $f(\gamma(t))=\sum_{n=0}^{\infty} a_{n}\left(t-t_{0}\right)^{n}$ for every $t \in\left(t_{0}-\delta, t_{0}+\delta\right) \cap I$.

The following proposition associates the phenomenon of real analyticity and that of holomorphically extendability.

Proposition 2.9 Let $\gamma: I \rightarrow \mathbb{C}$ be an analytic curve at $t_{0}$ and $t_{0} \in I$. A function $f: \gamma^{*} \rightarrow \mathbb{C}$ is real analytic at $\left(t_{0}, z_{0}=\gamma\left(t_{0}\right)\right)$ if and only if $f$ is holomorphically extendable at $\left(t_{0}, z_{0}=\gamma\left(t_{0}\right)\right)$.

Proof At first we will prove direction $\Rightarrow:$. If $f$ is real analytic at $\left(t_{0}, z_{0}=\gamma\left(t_{0}\right)\right)$, then from Lemma 2.6

$$
f(\gamma(t))=\sum_{n=0}^{\infty} b_{n}\left(\gamma(t)-\gamma\left(t_{0}\right)\right)^{n}
$$

for every $t \in\left(t_{0}-\epsilon, t_{0}+\epsilon\right) \cap I$ and for some $b_{n} \in \mathbb{C}, \epsilon>0$. From the continuity of $\gamma$, there exists $\eta>0$ such that $\gamma\left(\left(t_{0}-\eta, t_{0}+\eta\right) \cap I\right) \subset D\left(z_{0}, \epsilon\right)$. Therefore the function

$$
F(z)=\sum_{n=0}^{\infty} b_{n}\left(z-\gamma\left(t_{0}\right)\right)^{n}
$$

defined on $D\left(z_{0}, \epsilon\right)$ is equal to $f$ on $\gamma\left(\left(t_{0}-\eta, t_{0}+\eta\right) \cap I\right)$. Thus the function $f$ is holomorphically extendable at $\left(t_{0}, z_{0}=\gamma\left(t_{0}\right)\right)$.

Next we prove direction $\Leftarrow$ : If $f$ is extendable at $\left(t_{0}, z_{0}=\gamma\left(t_{0}\right)\right)$, then there are $r>0$ and a holomorphic function $F: D\left(\gamma\left(t_{0}\right), r\right) \rightarrow \mathbb{C}$ such that

$$
f(\gamma(t))=F(\gamma(t))
$$

for every $t \in\left(t_{0}-\epsilon, t_{0}+\epsilon\right) \cap I$ and for some $\epsilon>0$. Let

$$
\sum_{n=0}^{\infty} b_{n}\left(z-\gamma\left(t_{0}\right)\right)^{n}
$$

be the Taylor expansion of the holomorphic function $F$. It follows that

$$
f(\gamma(t))=F(\gamma(t))=\sum_{n=0}^{\infty} b_{n}\left(\gamma(t)-\gamma\left(t_{0}\right)\right)^{n}
$$

for every $t \in\left(t_{0}-\epsilon, t_{0}+\epsilon\right) \cap I$ and as a result, again from Lemma 2.6, we conclude that $f$ is real analytic at $\left(t_{0}, z_{0}=\gamma\left(t_{0}\right)\right)$, because the curve $\gamma$ is analytic at $t_{0}$. 
The following theorem is a consequence of Lemma 2.4 and Proposition 2.9.

Theorem 2.10 Let $\gamma: I \rightarrow \mathbb{C}$ be a continuous and locally injective curve and $t_{0} \in I$. Then $\gamma$ is analytic at $t_{0}$ if and only if for every function $f: \gamma^{*} \rightarrow \mathbb{C}$ the following are equivalent:

1. $f$ is real analytic at $\left(t_{0}, z_{0}=\gamma\left(t_{0}\right)\right)$.

2. $f$ is holomorphically extendable at $\left(t_{0}, z_{0}=\gamma\left(t_{0}\right)\right)$.

Now we will examine a different kind of differentiability.

Definition 2.11 Let $\gamma: X \rightarrow \mathbb{C}$ be a continuous and injective curve. We define the derivative of a function $f: \gamma^{*} \rightarrow \mathbb{C}$ at $\gamma\left(t_{0}\right)$, where $t_{0} \in X$ by

$$
\frac{\mathrm{d} f}{\mathrm{~d} z}\left(\gamma\left(t_{0}\right)\right)=\lim _{t \rightarrow t_{0}} \frac{f(\gamma(t))-f\left(\gamma\left(t_{0}\right)\right)}{\gamma(t)-\gamma\left(t_{0}\right)}
$$

if the above limit exists and is a complex number. Inductively, we define

$$
\frac{\mathrm{d}^{k} f}{\mathrm{~d} z^{k}}(\gamma(t))=\frac{\mathrm{d}\left(\frac{\mathrm{d}^{k-1} f}{\mathrm{~d} z^{k-1}}(\gamma(t))\right)}{\mathrm{d} z}(\gamma(t)) .
$$

Definition 2.12 Let $\gamma: X \rightarrow \mathbb{C}$ be a homeomorphism onto $\gamma^{*}$. A function $f$ : $\gamma^{*} \rightarrow \mathbb{C}$ which belongs to the class $C^{k}\left(\gamma^{*}\right)$ if $\frac{\mathrm{d}^{k} f}{\mathrm{~d} z^{k}}(\gamma(t))$ exists and is continuous for $t \in X$. Finally, a function $f: \gamma^{*} \rightarrow \mathbb{C}$ belongs to the class $C^{\infty}\left(\gamma^{*}\right)$ if $\frac{\mathrm{d}^{k} f}{\mathrm{~d} z^{k}}(\gamma(t))$ exists and is continuous for every $t \in X$ and for every $k \in\{1,2,3, \ldots\}$. Let also $\left(X_{n}\right), n \in\{1,2,3, \ldots\}$ be an increasing sequence of compact subsets of $\gamma^{*}$ such that $\bigcup_{n=1}^{\infty} X_{n}=\gamma^{*}$. For $k \in\{1,2,3, \ldots\} \cup\{\infty\}$, the topology of the space $C^{k}\left(\gamma^{*}\right)$ is defined by the seminorms

$$
\sup _{z \in X_{n}}\left|\frac{\mathrm{d}^{l} f}{\mathrm{~d} z^{l}}(z)\right|, l=0,1,2, \ldots, k, n=1,2,3, \ldots
$$

In this way $C^{k}\left(\gamma^{*}\right)$ becomes a Banach space if $k<\infty$ and $I$ is compact. Otherwise, $C^{k}\left(\gamma^{*}\right)$ is a Fréchet space.

Proposition 2.13 Let $\gamma: X \rightarrow \mathbb{C}$ be a homeomorphism onto $\gamma^{*}$ with $\gamma^{\prime}(t) \neq 0$ for every $t \in X$ and $k \in\{1,2,3, \ldots\} \cup\{\infty\}$. If $C^{k}(X)=C^{k}\left(\gamma^{*}\right) \circ \gamma$, then $\gamma \in C^{k}(X)$.

Proof The function $f: \gamma^{*} \rightarrow \mathbb{C}$ with $f(\gamma(t))=\gamma(t)$ for $t \in X$ belongs to the class $C^{k}\left(\gamma^{*}\right)$ and therefore the function $\gamma=f \circ \gamma: X \rightarrow \mathbb{C}$ belongs to the class $C^{k}(X)$.

Now we will prove the converse of the previous proposition. If $\gamma \in C^{k}(X)$, then $C^{k}(X)=C^{k}\left(\gamma^{*}\right) \circ \gamma$. In order to do so we need the following lemma which will also be useful to us later. Its proof is straightforward and thus omitted, but the interested reader can find a proof in [1]. 
Lemma 2.14 Let $X$ be an interval $I \subset \mathbb{R}$ or the unit circle $T, \gamma \in C^{k}(X), k \in$ $\{1,2, \ldots\} \cup\{\infty\}$ and $f \in C^{k}\left(\gamma^{*}\right)$ and $g=f \circ \gamma$. Then $g \in C^{k}(X)$ and there exist polynomials $P_{j, i}$ defined on $\mathbb{C}^{i}$, such that

$$
g^{(i)}(t)=\sum_{j=1}^{i} \frac{d^{j} f}{\mathrm{~d} z^{j}}(\gamma(t)) P_{j, i}\left(\gamma^{\prime}(t), \gamma^{\prime \prime}(t), \ldots, \gamma^{(i)}(t)\right)
$$

where the derivatives of $\gamma$ are with respect to the real variable $t$ in the case $X=I$ and with respect to the complex variable $t,|t|=1$ in the case $X=T$.

Proposition 2.15 Let $\gamma: X \rightarrow \mathbb{C}$ be a homeomorphism onto $\gamma^{*}$ with $\gamma^{\prime}(t) \neq 0$ and $k \in\{1,2,3, \ldots\} \cup\{\infty\}$. If $\gamma \in C^{k}(X)$, then $C^{k}(X)=C^{k}\left(\gamma^{*}\right) \circ \gamma$.

Proof By Lemma 2.14 we have that $C^{k}(X) \supset C^{k}\left(\gamma^{*}\right) \circ \gamma$. We proceed by proving inductively that $C^{k}(X) \subset C^{k}\left(\gamma^{*}\right) \circ \gamma$.

For $k=1$ whenever $f \in C^{1}(X)$ we denote $g=f \circ \gamma^{-1}$. Then, it is easy to see that

$$
\frac{\mathrm{d} g}{\mathrm{~d} z}\left(\gamma\left(t_{0}\right)\right)=\frac{f^{\prime}\left(t_{0}\right)}{\gamma^{\prime}\left(t_{0}\right)}
$$

for $t_{0} \in X$ which implies that the function $\frac{d g}{\mathrm{~d} z}$ is continuous and hence $g \in C^{1}\left(\gamma^{*}\right)$.

Assume that the assertion holds for some $k$. Given $f \in C^{k+1}(X)$ we denote $g=$ $f \circ \gamma^{-1}$. Notice that $g \circ \gamma=f$ and

$$
\frac{\mathrm{d} g}{\mathrm{~d} z}\left(\gamma\left(t_{0}\right)\right)=\frac{\left(f^{\prime} \circ \gamma^{-1}\right)\left(\gamma\left(t_{0}\right)\right)}{\gamma^{\prime}\left(t_{0}\right)}
$$

for $t_{0} \in X$. From the induction hypothesis, we obtain that $f^{\prime} \circ \gamma^{-1} \in C^{k}\left(\gamma^{*}\right)$. It is also true that $\gamma^{\prime} \in C^{k}(X)$. It follows immediately that $\frac{\mathrm{d} g}{\mathrm{~d} z} \in C^{k}\left(\gamma^{*}\right)$ or equivalently $g \in C^{k+1}\left(\gamma^{*}\right)$ and the proof is complete for every finite $k$.

The case $k=\infty$ follows from the case of finite $k$.

As we have shown above if $\gamma$ is a homeomorphism defined on $X$, then $C(X)=$ $C\left(\gamma^{*}\right) \circ \gamma$. If also $\gamma \in C^{k}(X)$, where $k \in\{0,1,2, \ldots\} \cup\{\infty\}$, and $\gamma^{\prime}(t) \neq 0$ for every $t \in X$, then $C^{k}(X)=C^{k}\left(\gamma^{*}\right) \circ \gamma$. This implies that the spaces $C^{k}\left(\gamma^{*}\right)$ and $C^{k}(\gamma)$ contain exactly the same elements. Now we will prove that they also share the same topology.

Proposition 2.16 Let $\gamma$ be a homeomorphism defined on $X$ of class $C^{k}(X)$ and $\gamma^{\prime}(t) \neq 0$ for every $t \in X$. Then the spaces $C^{k}(\gamma)$ and $C^{k}\left(\gamma^{*}\right)$ share the same topology.

Proof At first we will prove the proposition in the special case where $X$ is a compact interval $I \subset \mathbb{R}$ or the unit circle $T$ and $k \neq \infty$. In order to do so we will find 
$0<M, N<\infty$ such that $d_{1}\left(f_{1}, f_{2}\right) \leq M d_{2}\left(f_{1}, f_{2}\right)$ and $d_{2}\left(f_{1}, f_{2}\right) \leq N d_{1}\left(f_{1}, f_{2}\right)$ for every $f_{1}, f_{2} \in C^{k}(\gamma)=C^{k}\left(\gamma^{*}\right)$, where $d_{1}, d_{2}$ are the metrics of $C^{k}(\gamma)$ and $C^{k}\left(\gamma^{*}\right)$, respectively.

Let $f_{1}, f_{2} \in C^{k}\left(\gamma^{*}\right)$ and $g_{1}=f_{1} \circ \gamma, g_{2}=f_{2} \circ \gamma \in C^{k}(X)$. We notice that

$$
\sup _{t \in X}\left|g_{1}(t)-g_{2}(t)\right|=\sup _{z \in \gamma^{*}}\left|f_{1}(z)-f_{2}(z)\right| \leq d_{2}\left(f_{1}, f_{2}\right) .
$$

In addition,

$$
\left(g_{1}-g_{2}\right)^{(i)}(t)=\sum_{j=1}^{i} \frac{d^{j}\left(f_{1}-f_{2}\right)}{\mathrm{d} z^{j}}(\gamma(t)) P_{j, i}\left(\gamma^{\prime}(t), \gamma^{\prime \prime}(t), \ldots, \gamma^{(i)}(t)\right),
$$

for $1 \leq j \leq i \leq k$, where $P_{j, i}$ are the polynomials of Lemma 2.14. If $m_{j, i}=$ $\sup _{i \in X} \mid P_{j, i}\left(\left(\gamma^{\prime}(t), \gamma^{\prime \prime}(t), \ldots, \gamma^{(i)}(t)\right) \mid\right.$ and $M_{i}=\max \left\{m_{j, i}, 1 \leq j \leq i\right\}$, then from the $t \in X$

triangle inequality

$$
\sup _{t \in X}\left|\left(g_{1}-g_{2}\right)^{(i)}(t)\right| \leq M_{i} d_{2}\left(f_{1}, f_{2}\right) .
$$

Consequently, $d_{1}\left(f_{1}, f_{2}\right) \leq M d_{2}\left(f_{1}, f_{2}\right)$, where $M=1+M_{1}+M_{2}+\cdots+M_{k}$.

We also notice that

$$
\sup _{z \in \gamma^{*}}\left|f_{1}(z)-f_{2}(z)\right|=\sup _{t \in X}\left|g_{1}(t)-g_{2}(t)\right| \leq d_{1}\left(f_{1}, f_{2}\right) .
$$

We will prove inductively that

$$
\sup _{z \in \gamma^{*}}\left|\frac{d^{i}\left(f_{1}-f_{2}\right)}{\mathrm{d} z^{i}}(z)\right| \leq N_{i} d_{1}\left(f_{1}, f_{2}\right)
$$

for some $N_{i}>0, i=1,2, \ldots, i \leq k$. It is easy to see that $P_{i, i}\left(z_{1}, z_{2}, \ldots, z_{i}\right)=z_{1}{ }^{i}$. Moreover, $\gamma^{\prime}(t) \neq 0$ for every $t \in X$ implies

$$
s_{i}=\inf _{t \in X} \mid P_{i, i}\left(\left(\gamma^{\prime}(t), \gamma^{\prime \prime}(t), \ldots, \gamma^{(i)}(t)\right) \mid>0\right.
$$

. For $i=1 \leq k$ we have

$$
\sup _{z \in \gamma^{*}}\left|\frac{d\left(f_{1}-f_{2}\right)}{\mathrm{d} z}(z)\right| \leq \frac{1}{s_{1}} \sup _{t \in X}\left|\left(g_{1}-g_{2}\right)^{\prime}(t)\right| \leq \frac{1}{s_{1}} d_{1}\left(f_{1}, f_{2}\right),
$$


and thus $N_{1}=\frac{1}{s_{1}}$. Assume that the assertion holds for every $1 \leq j \leq i<k$. Using our induction hypothesis and equation (1), we find from the reverse triangle inequality

$$
\sup _{t \in X}\left|\left(g_{1}-g_{2}\right)^{(i+1)}(t)\right| \geq \sup _{z \in \gamma^{*}}\left|\frac{d^{i+1}\left(f_{1}-f_{2}\right)}{\mathrm{d} z^{i+1}}(z)\right| s_{i+1}-\sum_{j=2}^{i} N_{j} m_{j, i+1} d_{1}\left(f_{1}, f_{2}\right) .
$$

Therefore

$$
\sup _{z \in \gamma^{*}}\left|\frac{d^{i+1}\left(f_{1}-f_{2}\right)}{\mathrm{d} z^{i+1}}(z)\right| \leq N_{i+1} d_{1}\left(f_{1}, f_{2}\right)
$$

where $N_{i+1}=\frac{1}{s_{i+1}}+\sum_{j=2}^{i} \frac{N_{j} m_{j, i+1}}{s_{i+1}}$ and the result holds also for $i+1$. The induction is complete. Now it is easy to deduce that $d_{2}\left(f_{1}, f_{2}\right) \leq N d_{1}\left(f_{1}, f_{2}\right)$, where $N=$ $1+N_{1}+N_{2}+\cdots+N_{k}$.

It follows immediately from the above that even in the case where $X$ is any type of interval $I \subset \mathbb{R}$ and/or $k=\infty$ the respective topologies of the spaces $C^{k}(\gamma)$ and $C^{k}\left(\gamma^{*}\right)$ are the same. The basic open subsets of $C^{k}(\gamma)$ are defined by a compact subset of $X$, an $l \in\{0,1,2, \ldots\}, l \leq k$, a function $f \in C^{k}(\gamma)$, and an $\varepsilon>0$. But if we recall the definition of the topology of $C^{k}\left(\gamma^{*}\right)$ and use the above we realize that this basic open subset of $C^{k}(\gamma)$ is also an open subset of $C^{k}\left(\gamma^{*}\right)$. Similarly every basic open subset of $C^{k}\left(\gamma^{*}\right)$ is an open subset of $C^{k}(\gamma)$. The proof is complete.

Combining Propositions 2.13, 2.15, and 2.16, we obtain the following theorem.

Theorem 2.17 Let $\gamma: X \rightarrow \mathbb{C}$ be a homeomorphism onto $\gamma^{*}$ with $\gamma^{\prime}(t) \neq 0$ for every $t \in X$ and $k \in\{1,2,3, \ldots\} \cup\{\infty\}$. Then $C^{k}(X)=C^{k}\left(\gamma^{*}\right) \circ \gamma$ if and only if $\gamma \in C^{k}(X)$. In addition, the spaces $C^{k}(\gamma)$ and $C^{k}\left(\gamma^{*}\right)$ share the same topology.

Remark 2.18 One can prove a slightly stronger statement than that of Theorem 2.17. We need not assume $\gamma^{\prime}(t) \neq 0$. Then $C^{k}(X)=C^{k}\left(\gamma^{*}\right) \circ \gamma$ if and only if $\gamma \in C^{k}(X)$ and $\gamma^{\prime}(t) \neq 0$ for every $t \in X$.

Remark 2.19 With the definition of the derivative as in the Definition 2.12, we can define the spaces $C^{k}(E)$ for more general sets $E \subset \mathbb{C}$ but it may occur that the space $C^{k}(E)$ is not complete.

Theorem 2.17 shows that if $\gamma$ is a homeomorphism and $\gamma^{\prime}(t) \neq 0$ for $t \in X$, then $C^{k}(\gamma) \approx C^{k}\left(\gamma^{*}\right)$. Therefore, we obtain the following corollary.

Corollary 2.20 Let $\gamma: X \rightarrow \mathbb{C}$ be a homeomorphism onto $\gamma^{*}$ with $\gamma^{\prime}(t) \neq 0$ for every $t \in X$ and $k \in\{1,2,3, \ldots\} \cup\{\infty\}$. Then the space $C^{k}(\gamma)$ is independent of the parametrization of $\gamma$ and coincides with the space $C^{k}\left(\gamma^{*}\right)$. 


\section{Continuous Analytic Capacities}

In this section, we present a few facts for the notion of continuous analytic capacity [7] that we will need in Sects. 5 and 7. Section 7 leads us to generalize this notion and thus define the notion of $p$-continuous analytic capacity.

Definition 3.1 Let $U \subset \mathbb{C}$ be open. A function $f$ belongs to the class $A(U)$ if $f \in$ $H(U)$ and $f$ has a continuous extension on $\bar{U}$, where the closure of $U$ is taken in $\mathbb{C}$.

Definition 3.2 Let $\Omega$ be the complement in $\mathbb{C}$ of a compact set. A function $f$ belongs to the class $A(\Omega \cup\{\infty\})$ if $f \in H(\Omega) \cap C(\Omega \cup\{\infty\})$ and $f$ has a continuous extension on $\bar{\Omega}$, where the closure of $\Omega$ is taken in $\mathbb{C}$.

By Tietze's extension Theorem, the extensions in both previous definitions can be considered as extensions on the whole of $\mathbb{C} \cup\{\infty\}$ without increase of the original norm $\|f\|_{\infty}$.

Definition 3.3 Let $L$ be a compact subset of $\mathbb{C}$. Let also $\Omega=\mathbb{C} \backslash L$. We denote

$$
a(L)=\sup \left\{\left|\lim _{z \rightarrow \infty} z(f(z)-f(\infty))\right|: f \in A(\Omega \cup\{\infty\}),\|f\|_{\infty} \leq 1\right\}
$$

the continuous analytic capacity of $L$.

It is well known [7] that $a(L)=0$ if and only if $A(\Omega \cup\{\infty\})$ contains only constant functions.

Theorem 3.4 Let $L$ be a compact subset of $\mathbb{C}$ and $U \subset \mathbb{C}$ be open with $L \subset U$. Then $a(L)=0$ if and only if every $f \in C(U) \cap H(U \backslash L)$ belongs to $H(U)$.

Proof Assume that every $f \in C(U) \cap H(U \backslash L)$ belongs to $H(U)$.

We consider an arbitrary $f \in A(\Omega \cup\{\infty\})$. Since $f$ can be continuously extended over $L$, it belongs to $C(U) \cap H(U \backslash L)$ and thus to $H(U)$. Therefore, $f$ is analytic in $\mathbb{C}$ and continuous in $\mathbb{C} \cup\{\infty\}$ and hence it is constant.

Thus $a(L)=0$.

Now assume $a(L)=0$ and we consider any $f \in C(U) \cap H(U \backslash L)$.

There exist two closed curves $\gamma_{1}$ and $\gamma_{2}$ in $U$ so that $\gamma_{1}$ surrounds $L$ and $\gamma_{2}$ surrounds $\gamma_{1}$. We define the analytic functions

$$
\phi_{1}(z)=\frac{1}{2 \pi i} \int_{\gamma_{1}} \frac{f(\zeta)}{\zeta-z} \mathrm{~d} \zeta \quad \text { for } z \text { in the exterior of } \gamma_{1}
$$

and

$$
\phi_{2}(z)=\frac{1}{2 \pi i} \int_{\gamma_{2}} \frac{f(\zeta)}{\zeta-z} \mathrm{~d} \zeta \quad \text { for } z \text { in the interior of } \gamma_{2} \text {. }
$$

Then the function $g$ which equals $\phi_{2}-f$ in the interior of $\gamma_{2}$ and $\phi_{1}$ in the exterior of $\gamma_{1}$ is well defined and belongs to $A(\Omega \cup\{\infty\})$. Therefore $g$ is constant and thus $f$ is analytic in the interior of $\gamma_{2}$. Hence $f \in H(U)$. 
Due to the local nature of the proof of the next theorem, we shall state a few facts about the so-called Vitushkin's localization operator [5].

Let $U \subset \mathbb{C}$ be open and $f \in C(\mathbb{C}) \cap H(U)$. Let also $g \in C^{1}(\mathbb{C})$ have compact support. We define the function

$$
\begin{aligned}
G(z) & =\frac{1}{\pi} \iint \frac{f(z)-f(w)}{z-w} \frac{\partial g}{\partial \bar{w}}(w) \mathrm{d} m(w) \\
& =f(z) g(z)-\frac{1}{\pi} \iint \frac{f(w)}{z-w} \frac{\partial g}{\partial \bar{w}}(w) \mathrm{d} m(w) .
\end{aligned}
$$

The function $G$ is continuous in $\mathbb{C} \cup\{\infty\}$ with $G(\infty)=0$, analytic in $U \cup(\mathbb{C} \backslash \operatorname{supp} g)$ and $f-G$ is analytic in the interior of the set $g^{-1}(\{1\})$.

Definition 3.5 Let $L$ be a closed subset of $\mathbb{C}$. We define

$$
a(L)=\sup \{a(M): M \text { compact subset of } L\}
$$

the continuous analytic capacity of $L$.

Theorem 3.6 Let $L$ be a closed subset of $\mathbb{C}$. Then $a(L)=0$ if and only if for every open set $U \subset \mathbb{C}$ every $f \in C(U) \cap H(U \backslash L)$ belongs to $H(U)$.

Proof One direction is immediate from Theorem 3.4 and Definition 3.5 and hence we assume that $a(L)=0$.

We consider an arbitrary open set $U \subset \mathbb{C}$ which intersects $L$ and an arbitrary $f \in$ $C(U) \cap H(U \backslash L)$ and we shall prove that $f$ extends analytically over $U \cap L$.

Now $L$ may not be contained in $U$ but since analyticity is a local property we shall employ Vitushkin's localization operator.

Let $z_{0} \in U \cap L$ and $\overline{D\left(z_{0}, 3 \delta\right)} \subset U$. We consider $g \in C^{1}(\mathbb{C})$ with supp $g \subset \overline{D\left(z_{0}, 2 \delta\right)}$ such that $g=1$ in $\overline{D\left(z_{0}, \delta\right)}$.

We also consider the restriction $F$ of $f$ in $\overline{D\left(z_{0}, 3 \delta\right)}$ and we extend $F$ so that it is continuous in $\mathbb{C} \cup\{\infty\}$.

We define as in (2) the function

$$
G(z)=\frac{1}{\pi} \iint \frac{F(z)-F(w)}{z-w} \frac{\partial g}{\partial \bar{w}}(w) \mathrm{d} m(w) .
$$

Now $G$ is continuous in $\mathbb{C} \cup\{\infty\}$ with $G(\infty)=0$, analytic in $\left(D\left(z_{0}, 3 \delta\right) \backslash L\right) \cup(\mathbb{C} \backslash$ $\left.D\left(z_{0}, 2 \delta\right)\right)=\mathbb{C} \backslash\left(\overline{D\left(z_{0}, 2 \delta\right)} \cap L\right)$ and $f-G=F-G$ is analytic in $D\left(z_{0}, \delta\right)$.

Since $a(L)=0$, we have $a\left(\overline{D\left(z_{0}, 2 \delta\right)} \cap L\right)=0$ and hence $G$ is constant 0 in $\mathbb{C}$. Therefore, $f$ is analytic in $D\left(z_{0}, \delta\right)$.

Since $z_{0} \in U \cap L$ is arbitrary we conclude that $f \in H(U)$.

Theorem 3.7 [7] If $L$ is a Jordan arc with locally finite length, then $a(L)=0$. The same holds for any countable union of such curves. Therefore, line segments, circular arcs, analytic curves, and boundaries of convex sets are all of zero continuous analytic capacity. 
Definition 3.8 Let $U$ be an open subset of $\mathbb{C}$ and $p \in\{0,1,2, \ldots\} \cup\{\infty\}$. A function $f$ belongs to the class $A^{p}(U)$ if $f \in H(U)$ and all derivatives $f^{(j)}, j \in\{0,1,2, \ldots\}$, $0 \leq j \leq p$, have continuous extensions $f^{(j)}$ on $\bar{U}$, where the closure of $U$ is taken in $\mathbb{C}$.

Definition 3.9 Let $\Omega$ be the complement in $\mathbb{C}$ of a compact set and $p \in\{0,1,2, \ldots\} \cup$ $\{\infty\}$. A function $f$ belongs to the class $A^{p}(\Omega \cup\{\infty\})$ if $f \in H(\Omega) \cap C(\Omega \cup\{\infty\})$ and all derivatives $f^{(j)}, j \in\{0,1,2, \ldots\}, 0 \leq j \leq p$, have continuous extensions $f^{(j)}$ on $\bar{\Omega}$, where the closure of $\Omega$ is taken in $\overline{\mathbb{C}}$.

Definition 3.10 Let $L$ be a compact subset of $\mathbb{C}$ and $p \in\{0,1,2, \ldots\} \cup\{\infty\}$. Let also $\Omega=\mathbb{C} \backslash L$. For $p \neq \infty$ we denote the $p$-continuous analytic capacity as

$a_{p}(L)=\sup \left\{\left|\lim _{z \rightarrow \infty} z(f(z)-f(\infty))\right|: f \in A^{p}(\Omega \cup\{\infty\}), \max _{0 \leq j \leq p}\left\|f^{(j)}\right\|_{\infty} \leq 1\right\}$.

Obviously, $a_{0}(L)=a(L)$.

For $p=\infty$,

$$
a_{\infty}(L)=\sup \left\{\left|\lim _{z \rightarrow \infty} z(f(z)-f(\infty))\right|: f \in A^{\infty}(\Omega \cup\{\infty\}), d(f, 0) \leq 1\right\},
$$

where the Fréchet distance $d(f, 0)$ is defined by

$$
d(f, 0)=\sum_{j=0}^{\infty} 2^{-j} \frac{\left\|f^{(j)}\right\|_{\infty}}{1+\left\|f^{(j)}\right\|_{\infty}} .
$$

If $L$ is a closed subset of $\mathbb{C}$, then we define

$$
a_{p}(L)=\sup \left\{a_{p}(M): M \text { compact subset of } L\right\} .
$$

Definition 3.11 Let $L$ be a compact subset of $\mathbb{C}$ and $p \in\{0,1,2, \ldots\} \cup\{\infty\}$. Let also $\Omega=\mathbb{C} \backslash L$. We denote

$$
a_{p}^{\prime}(L)=\sup \left\{\left|\lim _{z \rightarrow \infty} z(f(z)-f(\infty))\right|: f \in A^{p}(\Omega \cup\{\infty\}),\|f\|_{\infty} \leq 1\right\} .
$$

Obviously, $a_{0}^{\prime}(L)=a(L)$.

If $L$ is a closed subset of $\mathbb{C}$ then we define

$$
a_{p}^{\prime}(L)=\sup \left\{a_{p}^{\prime}(M): M \text { compact subset of } L\right\} .
$$

It is obvious that $a_{p}(L)$ and $a_{p}^{\prime}(L)$ are decreasing functions of $p$.

The following theorem corresponds to Theorem 3.4.

Theorem 3.12 Let $L$ be a compact subset of $\mathbb{C}$ and $U \subset \mathbb{C}$ be open with $L \subset U$ and let $p \in\{0,1,2, \ldots\} \cup\{\infty\}$. Then $a_{p}(L)=0$ if and only if every $f \in A^{p}(U \backslash L)$ has an extension to $A^{p}(U)$, if and only if $a_{p}^{\prime}(L)=0$. 
The proof is a repetition of the proof of Theorem 3.4.

Definition 3.13 Let $L$ be a compact subset of $\mathbb{C}$. For $p \in\{0,1,2, \ldots\} \cup\{\infty\}$, we define $b_{p}(L)$ such that $b_{p}(L)=0$ when $a_{p}(L)=0$ and $b_{p}(L)=\infty$ when $a_{p}(L) \neq 0$. For $p, q \in\{0,1,2, \ldots\} \cup\{\infty\}$ we will say that $a_{p}(L)$ and $a_{q}(L)$ are essentially different if $b_{p}(L) \neq b_{q}(L)$.

Definition 3.14 Let $U$ be an open subset of $\mathbb{C}$ and $p \in\{1,2, \ldots\} \cup\{\infty\}$. A function $f$ belongs to the class $\tilde{A}^{p}(U)$ if $f \in A^{p}(U)$ and for $0 \leq j \leq j^{\prime} \leq p$ the following is true for $z, w \in \bar{U}$

$$
f^{(j)}(w)-\sum_{k=0}^{j^{\prime}-j} \frac{1}{k !} f^{(j+k)}(z)(w-z)^{k}=o\left(|w-z|^{j^{\prime}-j}\right) \quad \text { as } w \rightarrow z .
$$

This is supposed to hold uniformly for $z, w$ in compact subsets of $\bar{U}$.

Analogously, if $\Omega$ is the complement in $\mathbb{C}$ of a compact set, then a function $f$ belongs to the class $\tilde{A}^{p}(\Omega \cup\{\infty\})$ if $f \in A^{p}(\Omega \cup\{\infty\})$, and (3) holds for $z, w \in \bar{\Omega}$.

Note that, since $f \in H(U)$, relation (3) is automatically true for $z \in U$ and thus the "point" of the definition is when $z \in \partial U$.

If $p$ is finite, then $f \in \tilde{A}^{p}(U)$ admits as a norm $\|f\|_{\tilde{A}^{p}(U)}$ the smallest $M$ such that

$$
\begin{aligned}
& \left|f^{(j)}(z)\right| \leq M \quad \text { for } z \in \bar{U}, 0 \leq j \leq p \\
& \left|f^{(j)}(w)-\sum_{k=0}^{j^{\prime}-j} \frac{1}{k !} f^{(j+k)}(z)(w-z)^{k}\right| \leq M|w-z|^{j^{\prime}-j} w, z \in \bar{U},|w-z| \leq 1, \\
& 0 \leq j \leq j^{\prime} \leq p
\end{aligned}
$$

It is easy to see that $\tilde{A}^{p}(U)$ with this norm is complete.

If $p$ is infinite, then using the norms for the finite cases in the standard way, $\tilde{A}^{\infty}(U)$ becomes a Fréchet space.

There is a fundamental result of Whitney [13] saying that if $f \in \tilde{A}^{p}(U)$, then $f$ can be extended in $\mathbb{C}$ in such a way that the extended $f$ belongs to $C^{p}(\mathbb{C})$ and that the partial derivatives of $f$ of order $\leq p$ in $\mathbb{C}$ are extensions of the original partial derivatives of $f$ in $U$.

Definition 3.15 Let $L$ be a compact subset of $\mathbb{C}$ and $p \in\{0,1,2, \ldots\} \cup\{\infty\}$. Let also $\Omega=\mathbb{C} \backslash L$. For $p \neq \infty$, we denote

$$
\tilde{a}_{p}(L)=\sup \left\{\left|\lim _{z \rightarrow \infty} z(f(z)-f(\infty))\right|: f \in \tilde{A}^{p}(\Omega \cup\{\infty\}),\|f\|_{\tilde{A}^{p}(U)} \leq 1\right\} .
$$

In the case $p=\infty$, the norm $\|f\|_{\tilde{A}^{p}(U)}$ is replaced by the distance from $f$ to 0 in the metric space structure of $\tilde{A}^{\infty}(U)$.

Obviously, $\tilde{a}_{0}(L)=a(L)$. 
If $L$ is a closed subset of $\mathbb{C}$, then we define

$$
\tilde{a}_{p}(L)=\sup \left\{\tilde{a}_{p}(M): M \text { compact subset of } L\right\} .
$$

It turns out that in the case $p \geq 1$, there is a simple topological characterization of the compact sets $L$ with $\tilde{a}_{p}(L)=0$.

Theorem 3.16 Let $L$ be a compact subset of $\mathbb{C}$ and $p \geq 1$. Then $\tilde{a}_{p}(L)=0$ if and only if L has empty interior.

Proof Let $L$ have non-empty interior and let the disk $D\left(z_{0}, r_{0}\right)$ be contained in $L$. Obviously, the non-constant function $\frac{1}{z-z_{0}}$ belongs to $\tilde{A}^{p}(\Omega \cup\{\infty\})$ for all $p$ and thus $\tilde{a}_{p}(L)>0$ for all $p$.

Conversely, let $L$ have empty interior and let $f$ belong to $\tilde{A}^{1}(\Omega \cup\{\infty\})$. Then $f$ is analytic in $\Omega$ and at every $z \in L$, we have

$$
f(w)-f(z)-f^{\prime}(z)(w-z)=o(|w-z|) \quad \text { as } w \rightarrow z, w \in \bar{\Omega}=\mathbb{C} .
$$

Thus $f$ is analytic at $z$ (with derivative equal to $f^{\prime}(z)$ ) and hence analytic in all of $\mathbb{C}$. Since $f$ is continuous at $\infty$, it is a constant. Therefore, $\tilde{A}^{1}(\Omega \cup\{\infty\})$ contains only the constant functions and $\tilde{a}_{1}(L)=0$.

Theorem 3.17 There is a compact subset $L$ of $\mathbb{C}$ such that $\tilde{a}_{1}(L)=0<\tilde{a}_{0}(L)$.

Proof Due to the last theorem, it is enough to find a compact $L$ with empty interior and with $\tilde{a}_{0}(L)=a(L)>0$.

This set $L$ is a Cantor type set. We consider a sequence $\left(a_{n}\right)$ with $0<a_{n}<\frac{1}{2}$ for every $n=1,2,3, \ldots$ and construct a sequence $\left(L_{n}\right)$ of decreasing compact sets as follows. $L_{0}$ is the unit square $[0,1] \times[0,1]$ and $L_{1}$ is the union of the four squares at the four corners of $L_{0}$ with side length equal to $a_{1}$. We then continue inductively. If $L_{n}$ is the union of $4^{n}$ squares each of sidelength equal to

$$
l_{n}=a_{1} \cdots a_{n},
$$

then each of these squares produces four squares at its four corners each of side length equal to $a_{1} \cdots a_{n} a_{n+1}$. The union of these new squares is $L_{n+1}$.

We denote $I_{n, k}, k=1, \ldots, 4^{n}$, the squares whose union is $L_{n}$.

Finally, we define

$$
L=\bigcap_{n=1}^{+\infty} L_{n}
$$

It is clear that $L$ is a totally disconnected compact set. The area of $L_{n}$ equals

$$
\left|L_{n}\right|=4^{n}\left(a_{1} \cdots a_{n}\right)^{2}=\left(2 a_{1} \cdots 2 a_{n}\right)^{2} .
$$


Now we assume that

$$
\sum_{n=1}^{+\infty}\left(1-2 a_{n}\right)<+\infty
$$

Under this condition, we find that the area of $L$ equals

$$
|L|=\lim _{n \rightarrow+\infty}\left|L_{n}\right|=\lim _{n \rightarrow+\infty}\left(2 a_{1} \cdots 2 a_{n}\right)^{2}>0 .
$$

Then it is well known [7] that the function

$$
f(z)=\frac{1}{\pi} \iint_{L} \frac{1}{z-w} \mathrm{~d} m(w)
$$

is continuous in $\mathbb{C} \cup\{\infty\}$ with $f(\infty)=0$ and holomorphic on $\mathbb{C} \backslash L$. Since

$$
\lim _{z \rightarrow \infty} z f(z)=|L|>0,
$$

$f$ is not identically equal to 0 and hence $\tilde{a}_{0}(L)=a(L)>0$.

Remark 3.18 The latter part of the above proof shows that if a compact set $L$ (not necessarily of Cantor type) has strictly positive area, then $a(L)>0$, which is a wellknown fact [7].

The problem of the characterization of the compact sets $L$ with $a_{p}(L)=0$ seems to be more complicated.

We will show that there is a compact set $L$ such that $a_{0}(L)$ and $a_{1}(L)$ are essentially different, i.e., $a_{0}(L)>0$ and $a_{1}(L)=0$.

Theorem 3.19 There is a compact subset $L$ of $\mathbb{C}$ such that $a_{1}(L)=0<a_{0}(L)$.

Proof We consider the same Cantor type set $L$ which appeared in the proof of the previous theorem. We keep the same notations.

We now take any $f$ which belongs to $A^{1}(\Omega \cup\{\infty\})$. Subtracting $f(\infty)$ from $f$, we may also assume that $f(\infty)=0$.

Let $z_{0} \in \Omega$. Then there is $n_{0}$ such that $z_{0} \notin L_{n}$ for all $n \geq n_{0}$.

By Cauchy's formula, for every $n \geq n_{0}$, we have

$$
f\left(z_{0}\right)=-\frac{1}{2 \pi i} \sum_{k=1}^{4^{n}} \int_{\gamma_{n, k}} \frac{f(z)}{z-z_{0}} \mathrm{~d} z
$$

where $\gamma_{n, k}$ is the boundary curve of the square $I_{n, k}$.

Let $z_{n, k}$ be any point of $\Omega$ inside $I_{n, k}$ (for example, the center of the square). It is geometrically obvious that for every $z \in \gamma_{n, k}$ there is a path (consisting of at most two 
line segments) $\gamma$ with length $l(\gamma) \leq 2 l_{n}$ joining $z$ and $z_{n, k}$ and contained in $\Omega$ (with the only exception of its endpoint $z)$. Since $f \in A^{1}(\Omega \cup\{\infty\})$, we get

$$
\begin{aligned}
& \left|f(z)-f\left(z_{n, k}\right)-f^{\prime}\left(z_{n, k}\right)\left(z-z_{n, k}\right)\right|=\left|\int_{\gamma}\left(f^{\prime}(\zeta)-f^{\prime}\left(z_{n, k}\right)\right) \mathrm{d} \zeta\right| \\
& \quad \leq \int_{\gamma}\left|f^{\prime}(\zeta)-f^{\prime}\left(z_{n, k}\right)\right| \mathrm{d} \zeta \leq \epsilon_{n} l(\gamma) \leq 2 \epsilon_{n} l_{n},
\end{aligned}
$$

where $\epsilon_{n} \rightarrow 0$ uniformly for $z \in \gamma_{n, k}$ and for $k=1, \ldots, 4^{n}$.

Therefore,

$$
\left|\int_{\gamma_{n, k}} \frac{f(z)}{z-z_{0}} \mathrm{~d} z\right|=\left|\int_{\gamma_{n, k}} \frac{f(z)-f\left(z_{n, k}\right)-f^{\prime}\left(z_{n, k}\right)\left(z-z_{n, k}\right)}{z-z_{0}} \mathrm{~d} z\right| \leq \epsilon_{n} \frac{8 l_{n}^{2}}{\delta_{0}},
$$

where $\delta_{0}$ is the distance of $z_{0}$ from $L_{n_{0}}$.

Thus from (4) we obtain

$$
\left|f\left(z_{0}\right)\right| \leq \frac{8 \cdot 4^{n} l_{n}^{2}}{\pi \delta_{0}} \epsilon_{n}=\frac{8\left|L_{n}\right|}{\pi \delta_{0}} \epsilon_{n} \leq \frac{8}{\pi \delta_{0}} \epsilon_{n} .
$$

This holds for all $n \geq n_{0}$ and hence $f\left(z_{0}\right)=0$ for all $z_{0} \in \Omega$.

We proved that the only element $f$ of $A^{1}(\Omega \cup\{\infty\})$ with $f(\infty)=0$ is the zero function and thus $a_{1}(L)=0$.

We will now see a different proof of the above theorem. The proof is longer from the previous one, but it provides a more general result.

Theorem 3.20 Let $K_{1 / 3}$ be the usual Cantor set lying on $[0,1]$ and $L=K_{1 / 3} \times K_{1 / 3}$. Then $a_{0}(L)>0$, but $a_{1}(L)=0$.

Proof It is known $[6,14]$ that there exists a function $g$ continuous on $S^{2}$ and holomorphic off $L$, such that

$$
g^{\prime}(\infty)=\lim _{z \rightarrow \infty} z(g(z)-g(\infty)) \neq 0
$$

which implies that $a_{0}(L)>0$.

For the second statement we first observe that the area of $L$ is 0 , as

$$
L=\bigcap_{n=0}^{\infty} L_{n}
$$

where each $L_{n}$ is the union of $4^{n}$ squares of area $9^{-n}$. We will prove that $a_{1}(L)=0$ or equivalently that every function in $A^{1}(\Omega \cup\{\infty\})$ is entire, where $\Omega=\mathbb{C} \backslash L$. Let $f \in A^{1}(\Omega \cup\{\infty\}), \varepsilon>0$, and $\varphi_{\varepsilon}=\varepsilon^{-2} \chi_{\varepsilon}$, where $\chi_{\varepsilon}$ is the characteristic function of the square $S_{\varepsilon}$ with center at 0 and sides parallel to the axes with length $\varepsilon$. It is easy to see from the continuity of $f$ that the convolutions $f * \varphi_{\varepsilon}$ belong to $C^{1}(\mathbb{C})$ and converge 
uniformly on $D(0,2)$ to $f$ as $\varepsilon \rightarrow 0$. Since $f \in A^{1}(\Omega \cup\{\infty\})$, the partial derivatives $\frac{\partial u}{\partial x}, \frac{\partial u}{\partial y}$ and $\frac{\partial v}{\partial x}, \frac{\partial v}{\partial y}$ of $u=\operatorname{Ref}$ and $v=\operatorname{Im} f$, respectively, extend continuously on $\mathbb{C} \cup\{\infty\}$ and hence are bounded. We will prove that

$$
\frac{\partial u * \varphi_{\varepsilon}}{\partial x}=\frac{\partial u}{\partial x} * \varphi_{\varepsilon}
$$

on $\Omega$. Let $(a, b) \in \Omega$ and $h \in \mathbb{R}^{*}$. Then

$$
\begin{aligned}
& \frac{\left(u * \varphi_{\varepsilon}\right)(a+h, b)-\left(u * \varphi_{\varepsilon}\right)(a, b)}{h} \\
& \quad=\varepsilon^{-2} \iint_{S_{\varepsilon}} \frac{u(a+h-x, b-y)-u(a-x, b-y)}{h} d x d y .
\end{aligned}
$$

It is easy to see that for almost every $(x, y) \in S_{\varepsilon}$ and every $h \in \mathbb{R}$, the segment $[(a+h-x, b-y),(a-x, b-y)]$ is a subset of $\Omega$. Thus from Mean Value Theorem for almost every $(x, y) \in S_{\varepsilon}$ and every $h \in \mathbb{R}^{*}$, there exists $q \in \mathbb{R}$, such that

$$
\frac{u(a+h-x, b-y)-u(a-x, b-y)}{h}=\frac{\partial u}{\partial x}(a+q-x, b-y),
$$

which remains bounded by a constant $M>0$. For those $(x, y) \in S_{\varepsilon}$

$$
\frac{u(a+h-x, b-y)-u(a-x, b-y)}{h}
$$

converges to

$$
\frac{\partial u}{\partial x}(a-x, b-y)
$$

as $h$ converges to 0 . Using the Dominated Convergence Theorem, we obtain (5). Similarly

$$
\frac{\partial u * \varphi_{\varepsilon}}{\partial y}=\frac{\partial u}{\partial y} * \varphi_{\varepsilon}, \frac{\partial v * \varphi_{\varepsilon}}{\partial x}=\frac{\partial v}{\partial x} * \varphi_{\varepsilon}, \frac{\partial v * \varphi_{\varepsilon}}{\partial y}=\frac{\partial v}{\partial y} * \varphi_{\varepsilon} .
$$

Since the Cauchy-Riemann equations are satisfied for $f$ almost everywhere, we have that

$$
\frac{\partial u}{\partial x} * \varphi_{\varepsilon}=\frac{\partial v}{\partial y} * \varphi_{\varepsilon}
$$

and

$$
\frac{\partial u}{\partial y} * \varphi_{\varepsilon}=-\frac{\partial v}{\partial x} * \varphi_{\varepsilon}
$$


Thus the Cauchy-Riemann equations are satisfied for every $f * \varphi_{\varepsilon}$ on $\Omega$. Since the interior of $L$ is void, the set $\Omega$ is dense in $\mathbb{C}$. From the continuity of the partial derivatives of every $f * \varphi_{\varepsilon}$ on $\mathbb{C}$, the Cauchy-Riemann equations are satisfied for every $f * \varphi_{\varepsilon}$ on $\mathbb{C}$, which implies that every $f * \varphi_{\varepsilon}$ is entire. Finally, $f * \varphi_{\varepsilon}$ converge uniformly on $D(0,2)$ to $f$, as $\varepsilon \rightarrow 0$, which implies that $f$ is holomorphic on $D(0,2)$ and hence entire.

Remark 3.21 The above proof also shows that if $L$ is a compact subset of $\mathbb{C}$ of zero area and if for almost every line $\varepsilon$ which is parallel to the $x$-axis and for almost every line $\varepsilon$ which is parallel to the $y$-axis, $\varepsilon \cap L=\emptyset$, then $a_{1}(L)=0$. In fact, it suffices that these intersections are finite for a dense set of $\varepsilon$ parallel to the $x$-axis and for a dense set of $\varepsilon$ parallel to the $y$-axis.

\section{Real Analyticity on Analytic Curves}

Let $L \subset \mathbb{C}$ be a closed set without isolated points. We denote by $C(L)$ the set of continuous functions $f$ on $L$. This space endowed with the topology of uniform convergence on the compact subsets of $L$ is a complete metric space and thus Baire's Theorem is at our disposal.

Lemma 4.1 Let $L \subset \mathbb{C}$ be a closed set without isolated points. Let also $z_{0} \in L$ be the center and $r>0$ be the radius of the open disk $D\left(z_{0}, r\right)$ and $M>0$. The set of functions $f \in C(L)$ for which there exists a holomorphic on $D\left(z_{0}, r\right)$ function $F$ bounded by $M$ such that $\left.F\right|_{D\left(z_{0}, r\right) \cap L}=\left.f\right|_{D\left(z_{0}, r\right) \cap L}$, is closed and has empty interior.

Proof Let $A\left(M, z_{0}, r\right)$ be the aforementioned set of functions. We distinguish two cases according to whether $D\left(z_{0}, r\right)$ is contained or not in $L$.

If $D\left(z_{0}, r\right) \subset L$, then the elements of $A\left(M, z_{0}, r\right)$ are holomorphic on $D\left(z_{0}, r\right)$ and bounded by $M$. Let $\left(f_{n}\right)_{n \geq 1}$ be a sequence in $A\left(M, z_{0}, r\right)$ converging uniformly on the compact subsets of $L$ to a function $f$ defined on $L$. Then $f$ is holomorphic on $D\left(z_{0}, r\right)$ and bounded by $M$ and $A\left(M, z_{0}, r\right)$ is a closed subset of $C(L)$.

For the second part of the theorem, let us assume that $A\left(M, z_{0}, r\right)$ does not have empty interior. Then there is a function $f$ in the interior of $A\left(M, z_{0}, r\right)$, a compact set $K \subset L$ and $\delta>0$ such that

$$
\mathcal{A}(f, K, \delta)=\left\{g \in C(L): \sup _{z \in K}|f(z)-g(z)|<\delta\right\} \subset A\left(M, z_{0}, r\right) .
$$

Then the function $h(z)=f(z)+\frac{\delta}{2} \bar{z}, z \in L$ belongs to $A\left(M, z_{0}, r\right)$ and therefore is holomorphic on $D\left(z_{0}, r\right)$. But then the function $\frac{\delta}{2} \bar{z}$ will be holomorphic on $D\left(z_{0}, r\right)$ which is absurd. Thus the interior of $A\left(M, z_{0}, r\right)$ is void.

If $D\left(z_{0}, r\right)$ is not contained in $L$, then there is $w \in D\left(z_{0}, r\right) \backslash L$. Let $\left(f_{n}\right)_{n \geq 1}$ be a sequence in $A\left(M, z_{0}, r\right)$ where $f_{n}$ converges uniformly on compact subsets of $L$ to a function $f$ defined on $L$. Then for every $n$ there are holomorphic functions $F_{n}: D\left(z_{0}, r\right) \rightarrow \mathbb{C}$ bounded by $M$ such that $\left.F_{n}\right|_{D\left(z_{0}, r\right) \cap L}=\left.f_{n}\right|_{D\left(z_{0}, r\right) \cap L}$. By Montel's 
Theorem, there is a subsequence $\left(F_{k_{n}}\right)$ of $\left(F_{n}\right)$ which converges uniformly to a function $F$ on the compact subsets of $D\left(z_{0}, r\right)$ which is holomorphic on $D\left(z_{0}, r\right)$ and bounded by $M$. Because $F_{k_{n}} \rightarrow f$ at $D\left(z_{0}, r\right) \cap L$ we have that $\left.F\right|_{D\left(z_{0}, r\right) \cap L}=\left.f\right|_{D\left(z_{0}, r\right) \cap L}$ and so $f \in A\left(M, z_{0}, r\right)$. Therefore $A\left(M, z_{0}, r\right)$ is a closed subset of $C(L)$.

If $A\left(M, z_{0}, r\right)$ does not have empty interior, then there exists a function $f$ in the interior of $A\left(M, z_{0}, r\right)$, a compact set $K \subset L$ and $\delta>0$ such that $\mathcal{A}(f, K, \delta) \subset$ $A\left(M, z_{0}, r\right)$. We choose $0<a<\delta \inf _{z \in K}|z-w|$. We notice that this is possible because $\inf _{z \in K}|z-w|>0$. The function $h(z)=f(z)+\frac{a}{2(z-w)}$ for $z \in L$ belongs to $A\left(M, z_{0}, r\right)$ and therefore has a holomorphic and bounded extension $H$ on $D\left(z_{0}, r\right)$, such that $\left.H\right|_{D\left(z_{0}, r\right) \cap L}=\left.h\right|_{D\left(z_{0}, r\right) \cap L}$. However, there is a holomorphic function $F$ : $D\left(z_{0}, r\right) \rightarrow \mathbb{C}$ which coincides with $f$ on $D\left(z_{0}, r\right) \cap L$. By analytic continuation $H(z)=F(z)+\frac{a}{2(z-w)}$ for $z \in D\left(z_{0}, r\right) \backslash\left\{z_{0}\right\}$, since they are equal on $L \cap$ $\left(D\left(z_{0}, r\right) \backslash\left\{z_{0}\right\}\right)$, which contains infinitely many points close to $z_{0}$, all of them being non-isolated. As a result, $H$ is not bounded at $D\left(z_{0}, r\right)$ which is a contradiction. Thus, $A\left(M, z_{0}, r\right)$ has empty interior.

Definition 4.2 Let $L \subset \mathbb{C}$ be a closed set without isolated points and $z_{0} \in L$. A function $f \in C(L)$ belongs to the class of non-holomorphically extendable at $z_{0}$ functions if there is no pair of an open disk $D\left(z_{0}, r\right), r>0$ and a holomorphic function $F: D\left(z_{0}, r\right) \rightarrow \mathbb{C}$ such that $\left.F\right|_{D\left(z_{0}, r\right) \cap L}=\left.f\right|_{D\left(z_{0}, r\right) \cap L}$.

Theorem 4.3 Let $L \subset \mathbb{C}$ be a closed set without isolated points and $z_{0} \in$ L. The class of non-holomorphically extendable at $z_{0}$ functions of $C(L)$ is dense and $G_{\delta}$.

Proof The set

$$
\bigcap_{n=1}^{\infty} \bigcap_{M=1}^{\infty}\left(C(L) \backslash A\left(M, z_{0}, \frac{1}{n}\right)\right)
$$

is a dense $G_{\delta}$ subset of $C(L)$ according to Baire's Theorem and coincides with the class of non-holomorphically extendable at $z_{0}$ functions, since every holomorphic function on $D\left(z_{0}, r\right)$ is bounded when restricted on $D\left(z_{0}, r^{\prime}\right)$ for $r^{\prime}<r$.

Definition 4.4 Let $L \subset \mathbb{C}$ be a closed set without isolated points. A function $f \in$ $C(L)$ belongs to the class of nowhere holomorphically extendable functions defined and continuous on $L$, if there exists no pair of an open disk $D\left(z_{0}, r\right), z_{0} \in L, r>0$ and a holomorphic function $F: D\left(z_{0}, r\right) \rightarrow \mathbb{C}$, such that $\left.F\right|_{D\left(z_{0}, r\right) \cap L}=\left.f\right|_{D\left(z_{0}, r\right) \cap L}$.

Theorem 4.5 Let $L \subset \mathbb{C}$ be a closed set without isolated points. The class of nowhere holomorphically extendable functions of $C(L)$ is a dense and $G_{\delta}$ subset of $C(L)$.

Proof Let $z_{l} \in L, l=1,2,3, \ldots$ be a dense sequence. Then the set

$$
\bigcap_{l=1}^{\infty} \bigcap_{n=1}^{\infty} \bigcap_{M=1}^{\infty}\left(C(L) \backslash A\left(M, z_{l}, \frac{1}{n}\right)\right)
$$


is a dense $G_{\delta}$ subset of $C(L)$ according to Baire's Theorem. This set coincides with the class of nowhere holomorphically extendable functions of $C(L)$, since every holomorphic function on $D\left(z_{0}, r\right)$ is bounded when restricted on $D\left(z_{0}, r^{\prime}\right)$ for $r^{\prime}<r$.

The proof of the above results can be used to prove similar results at some special cases. Let $\gamma: I \rightarrow \mathbb{C}$ be a continuous and locally injective curve, where $I$ is an interval in $\mathbb{R}$ of any type. The symbol $\gamma^{*}$ will be used instead of $\gamma(I)$. It is obvious that $\gamma^{*}$ has no isolated points.

Definition 4.6 Let $\gamma: I \rightarrow \mathbb{C}$ be a locally injective curve and $z_{0}=\gamma\left(t_{0}\right), t_{0} \in I$. A function $f: \gamma^{*} \rightarrow \mathbb{C}$ belongs to the class of non-holomorphically extendable at $\left(t_{0}, z_{0}=\gamma\left(t_{0}\right)\right)$ functions, if there are no open disk $D\left(z_{0}, r\right), r>0$, and $\eta>0$ and a holomorphic function $F: D\left(z_{0}, r\right) \rightarrow \mathbb{C}$, such that $\gamma\left(\left(t_{0}-\eta, t_{0}+\eta\right) \cap I\right) \subset D\left(z_{0}, r\right)$ and $F(\gamma(t))=f(\gamma(t))$ for all $t \in\left(t_{0}-\eta, t_{0}+\eta\right) \cap I$. Otherwise, $f$ is holomorphically extendable at $\left(t_{0}, z_{0}=\gamma\left(t_{0}\right)\right)$.

Theorem 4.7 Let $k, l \in\{0,1,2, \ldots\} \cup\{\infty\}$ such that $l \leq k$. Let also $\gamma: I \rightarrow \mathbb{C}$ be a locally injective function of $C^{k}(I)$ and $t_{0} \in I$. The class of non-holomorphically extendable at $\left(t_{0}, z_{0}=\gamma\left(t_{0}\right)\right)$ functions belonging to $C^{l}(\gamma)$ is a dense and $G_{\delta}$ subset of $C^{l}(\gamma)$.

Proof Let $r>0$ and $\eta>0$ such that $\gamma\left(t_{0}-\eta, t_{0}+\eta\right) \subset D\left(\gamma\left(t_{0}\right), r\right)$. Let also $A\left(M, z_{0}, r, \eta, l\right)$ be the set of $C^{l}(I)$ functions for which there is a holomorphic function $F$ defined on $D\left(z_{0}, r\right)$ and bounded by $M$, such that $F(\gamma(t))=f(\gamma(t))$ for every $t \in\left(t_{0}-\eta, t_{0}+\eta\right) \cap I$.

Since $\gamma \in C^{k}(I)$ the open disk $D\left(z_{0}, r\right)$ is not contained in $\gamma^{*}$ (see Proposition 6.2) and thus there is $w \in D\left(z_{0}, r\right) \backslash \gamma^{*}$. Similar to the proof of Lemma 4.1, $A\left(M, z_{0}, r, \eta, l\right)$ is a closed subset of $C^{l}(\gamma)$.

If $A\left(M, z_{0}, r, \eta, l\right)$ does not have empty interior, then there is a function $f$ in the interior of $A\left(M, z_{0}, r, \eta, l\right), b \in\{0,1,2, \ldots\}$, a compact set $K \subset I$, and $\delta>0$ such that

$\left\{g \in C^{k}(\gamma): \sup _{t \in K}\left|(f \circ \gamma)^{(j)}(t)-(g \circ \gamma)^{(j)}(t)\right|<\delta, 0 \leq j \leq b\right\} \subset A\left(M, z_{0}, r, \eta, l\right)$.

We choose $0<a<\delta \min \left\{\inf _{t \in K}|\gamma(t)-w|, \inf _{t \in K}|\gamma(t)-w|^{2}, \ldots, \frac{1}{b !} \inf _{t \in K}|\gamma(t)-w|^{b+1}\right\}$. This is possible because $w \notin \gamma^{*}$ and $\gamma(K) \subset \gamma^{*}$. The function $h(z)=f(z)+$ $\frac{a}{2(z-w)}$ for $z \in \gamma^{*}$ belongs to $A\left(M, z_{0}, r, \eta, l\right)$, since $\gamma \in C^{k}(I)$. Similar to the proof of Lemma 4.1, we are led to a contradiction. Therefore, $A\left(M, z_{0}, r, \eta, l\right)$ has empty interior.

Let $s_{n, m}, n, m=1,2,3, \ldots$ be a sequence such that $\lim _{m \rightarrow \infty} s_{n, m}=0$ for every $n$ and $\gamma\left(t_{0}-s_{n, m}, t_{0}+s_{n, m}\right) \subset D\left(\gamma\left(t_{0}\right), \frac{1}{n}\right)$ for every $n$ and every $m$. Then the class of non-holomorphically extendable at $\left(t_{0}, z_{0}=\gamma\left(t_{0}\right)\right)$ functions belonging to $C^{l}(\gamma)$ coincides with the set 


$$
\bigcap_{n=1}^{\infty} \bigcap_{m=1}^{\infty} \bigcap_{M=1}^{\infty}\left(C^{l}(\gamma) \backslash A\left(M, z_{0}, \frac{1}{n}, s_{n, m}, l\right)\right),
$$

because every holomorphic function on $D\left(z_{0}, r\right)$ becomes bounded if we restrict it on $D\left(z_{0}, r^{\prime}\right)$ for $r^{\prime}<r$. Thus according to Baire's Theorem, the class of nonholomorphically extendable at $\left(t_{0}, z_{0}=\gamma\left(t_{0}\right)\right)$ functions of $C^{l}(\gamma)$ is a dense and $G_{\delta}$ subset of $C^{l}(\gamma)$.

Definition 4.8 Let $\gamma: I \rightarrow \mathbb{C}$ be a locally injective curve. A function $f: \gamma^{*} \rightarrow \mathbb{C}$ belongs to the class of nowhere holomorphically extendable functions if there are no open disk $D\left(z_{0}, r\right), z_{0}=\gamma\left(t_{0}\right), t_{0} \in I, r>0$ and $\eta>0$ and a holomorphic function $F: D\left(z_{0}, r\right) \rightarrow \mathbb{C}$, such that $\gamma\left(\left(t_{0}-\eta, t_{0}+\eta\right) \cap I\right) \subset D\left(z_{0}, r\right)$ and $F(\gamma(t))=f(\gamma(t))$ for every $t \in\left(t_{0}-\eta, t_{0}+\eta\right) \cap I$.

Theorem 4.9 Let $k, l \in\{0,1,2, \ldots\} \cup\{\infty\}$ such that $l \leq k$. Let also $\gamma: I \rightarrow \mathbb{C}$ be a locally injective function of $C^{k}(I)$. The class of nowhere holomorphically extendable functions of $C^{l}(\gamma)$ is dense and $G_{\delta}$.

Proof Let $t_{n} \in I, n=1,2,3, \ldots$ be a dense sequence in $I$. Then the class of nowhere holomorphically extendable functions of $C^{k}(\gamma)$ coincides with the intersection over every $n$ of the classes of non-holomorphically extendable at $\left(t_{n}, z_{n}=\gamma\left(t_{n}\right)\right)$ functions of $C^{l}(\gamma)$. Since the classes of non-holomorphically extendable at $\left(t_{n}, z_{n}=\gamma\left(t_{n}\right)\right)$ functions of $C^{l}(\gamma)$ are dense and $G_{\delta}$ subsets of $C^{l}(\gamma)$ according to Theorem 4.7, it follows that the class of nowhere holomorphically extendable functions of $C^{l}(\gamma)$ is a dense and $G_{\delta}$ subset of $C^{l}(\gamma)$ from Baire's Theorem.

We intend to prove results about real analyticity using results about nonextendability. At first we notice that Proposition 2.9 and Theorem 4.9 immediately prove the following theorems.

Theorem 4.10 Let $\gamma: I \rightarrow \mathbb{C}$ be an analytic curve and $t_{0} \in I$. For $k=0,1,2, \ldots$ or $k=\infty$ the class of functions $f \in C^{k}(\gamma)$ which are not real analytic at $\left(t_{0}, z_{0}=\gamma\left(t_{0}\right)\right)$ is a dense and $G_{\delta}$ subset of $C^{k}(\gamma)$.

Theorem 4.11 Let $\gamma: I \rightarrow \mathbb{C}$ be an analytic curve. For $k=0,1,2, \ldots$ or $k=\infty$ the class of functions $f \in C^{k}(\gamma)$ which are nowhere real analytic is a dense and $G_{\delta}$ subset of $C^{k}(\gamma)$.

Remark 4.12 The fact that the class of functions $f \in C^{\infty}([0,1])$ which are nowhere real analytic is itself a dense and $G_{\delta}$ subset of $C^{\infty}([0,1])$ strengthens the result [3], where it is only proven that this class contains a dense and $G_{\delta}$ subset of $C^{\infty}([0,1])$.

Proposition 4.13 Let $\gamma: I \rightarrow \mathbb{C}$ be an analytic curve and $\gamma^{*}=\gamma(I)$. Let also $\Phi: \gamma^{*} \rightarrow \mathbb{C}$ be a homeomorphism of $\gamma^{*}$ on $\Phi\left(\gamma^{*}\right) \subset \mathbb{C}$ and $\delta=\Phi \circ \gamma$. Then the set of functions $f \in C^{k}(\delta), k \in\{0,1,2, \ldots\} \cup\{\infty\}$ which are nowhere analytic is a $G_{\delta}$ and dense subset of $C^{k}(\delta)$.

Proof The map $S: C^{k}(\gamma) \rightarrow C^{k}(\delta)$ defined by $S(g)=g \circ \Phi^{-1}, g \in C^{k}(\gamma)$ is a surjective isometry. Also a function $g \in C^{k}(\gamma)$ is nowhere analytic if and only if $S(g)$ is nowhere analytic. Theorem 4.11 combined with the above facts yields the result. 
Corollary 4.14 Assume that $J$ is an interval and $\gamma(t)=t$ or $J=\mathbb{R}$ and $\gamma(t)=e^{i t}$. Let $X$ denote the image of $\gamma$ and $\Phi: X \rightarrow \mathbb{C}$ be a homeomorphism of $X$ on $\Phi(X) \subset \mathbb{C}$ and $\delta=\Phi \circ \gamma$. Then the set of functions $f \in C^{k}(\delta), k \in\{0,1,2, \ldots\} \cup\{\infty\}$ which are nowhere analytic is a $G_{\delta}$ and dense subset of $C^{k}(\delta)$.

Proof The curve $\gamma$ is an analytic curve defined on an interval. The result follows from Proposition 4.13.

Remark 4.15 According to Corollary 4.14 for any Jordan curve or Jordan arc $\delta$ with a suitable parametrization generically on $C^{k}(\delta), k \in\{0,1,2, \ldots\} \cup\{\infty\}$ every function is nowhere analytic. In fact this holds for all parametrizations of $\delta^{*}$ and the spaces $C^{k}(\delta)$ are the same for all parametrizations so that $\delta$ is a homeomorphism between the unit circle $T$ or $[0,1]$ and $\delta^{*}$ (see Preliminaries).

\section{Extendability of Functions on Domains of Finite Connectivity}

We start this section with the following general fact. Its proof is skipped, but the interested reader can find one in [1].

Proposition 5.1 Let $n \in\{1,2, \ldots\}$. Let also $X_{1}, \ldots, X_{n}$ be complete metric spaces and $A_{1}, \ldots, A_{n}$ dense and $G_{\delta}$ subsets of $X_{1}, \ldots, X_{n}$, respectively. Then the metric space $X_{1} \times \cdots \times X_{n}$, endowed with the product topology, is complete and $A_{1} \times \cdots \times A_{n}$ is a dense and $G_{\delta}$ subset of $X_{1} \times \cdots \times X_{n}$.

Definition 5.2 Let $n \in\{1,2, \ldots\}$ and $\gamma_{i}: I_{i} \rightarrow \mathbb{C}, i=1, \ldots, n$ be continuous and locally injective curves, where $I_{i}$ are intervals. We define the space $C^{p_{1}, \ldots, p_{n}}\left(\gamma_{1}, \ldots, \gamma_{n}\right)=C^{p_{1}}\left(\gamma_{1}\right) \times \cdots \times C^{p_{n}}\left(\gamma_{n}\right)$, where $p_{i} \in\{0,1,2, \ldots\} \cup\{\infty\}$ for $i=1, \ldots, n$. The space $C^{p_{1}, \ldots, p_{n}}\left(\gamma_{1}, \ldots, \gamma_{n}\right)$ is endowed with the product topology and becomes a complete metric space.

We can regard the above space as the class of functions $f$, which are defined on the disjoint union $\gamma_{1}^{*} \cup \cdots \cup \gamma_{n}^{*}$ of the locally injective curves $\gamma_{1}, \ldots, \gamma_{n}$, where $\left.f\right|_{\gamma_{i}}$ belongs to $C^{p_{i}}\left(\gamma_{i}\right)$.

Definition 5.3 Let $n \in\{1,2, \ldots\}$ and $\gamma_{i}: I_{i} \rightarrow \mathbb{C}, i=1, \ldots, n$, be locally injective curves, where $I_{i}$ are intervals. A function $f$ defined on the disjoint union $\gamma_{1}^{*} \cup \cdots \cup \gamma_{n}^{*}$ for $z \in \gamma_{i}^{*}$ belongs to the class of nowhere holomorphically extendable functions if the restriction of $f$ on $\gamma_{i}^{*}$ belongs to the class of nowhere holomorphically extendable functions defined on $\gamma_{i}^{*}$, respectively, for every $i=1, \ldots, n$.

Theorem 5.4 Let $n \in\{1,2, \ldots\}, p_{i}, q_{i} \in\{0,1, \ldots\} \cup\{\infty\}$ such that $p_{i} \leq q_{i}$ for $i=1, \ldots, n$. Let also $\gamma_{i}: I_{i} \rightarrow \mathbb{C}, i=1, \ldots, n$, be locally injective functions of $C^{q_{i}}\left(I_{i}\right)$, where $I_{i}$ are intervals. The class of nowhere holomorphically extendable functions of $C^{p_{1}, \ldots, p_{n}}\left(\gamma_{1}, \ldots, \gamma_{n}\right)$ is a dense and $G_{\delta}$ subset of $C^{p_{1}, \ldots, p_{n}}\left(\gamma_{1}, \ldots, \gamma_{n}\right)$.

Proof Let $A_{i}$ be the class of nowhere holomorphically extendable functions of $C^{p_{i}}\left(\gamma_{i}\right)$. Then the set $A_{1} \times \cdots \times A_{n}$ coincides with the class of nowhere holomorphically extendable functions of $C^{p_{1}, \ldots, p_{n}}\left(\gamma_{1}, \ldots, \gamma_{n}\right)$. It follows from Theorem 4.9 that 
the sets $A_{1}, \ldots, A_{n}$ are dense and $G_{\delta}$ subsets of $C^{p_{1}}\left(\gamma_{1}\right), \ldots, C^{p_{n}}\left(\gamma_{n}\right)$, respectively, which combined with Proposition 5.1 implies that the class $A_{1} \times \cdots \times A_{n}$ is a dense and $G_{\delta}$ subset of $C^{p_{1}, \ldots, p_{n}}\left(\gamma_{1}, \ldots, \gamma_{n}\right)$.

Definition 5.5 Let $n \in\{1,2, \ldots\}$. Let also $\gamma_{i}: I_{i} \rightarrow \mathbb{C}, i=1, \ldots, n$, be locally injective curves, where $I_{i}$ are intervals. A function $f$ on the disjoint union $\gamma_{1}^{*} \cup \cdots \cup \gamma_{n}^{*}$, $f(z)=f_{i}(z)$ for $z \in \gamma_{i}^{*}$, is nowhere real analytic if the functions $f_{i}$ are nowhere real analytic for $i=1, \ldots, n$.

The proof of the following theorem is similar to the proof of Theorem 5.4.

Theorem 5.6 Let $n \in\{1,2, \ldots\}$ and $p_{i} \in\{0,1, \ldots\} \cup\{\infty\}, i=1, \ldots, n$. Let also $\gamma_{i}: I_{i} \rightarrow \mathbb{C}$ be analytic curves, where $I_{i}$ are intervals, $\Phi_{i}: \gamma_{i}^{*} \rightarrow \mathbb{C}$ be homeomorphisms of $\gamma_{i}^{*}$ on $\Phi_{i}\left(\gamma_{i}\right) \subset \mathbb{C}$ and let $\delta_{i}=\Phi_{i} \circ \gamma_{i}, i=1, \ldots, n$. The class of nowhere analytic functions $f \in C^{p_{1}, \ldots, p_{n}}\left(\delta_{1}, \ldots, \delta_{n}\right)$ is a dense and $G_{\delta}$ subset of $C^{p_{1}, \ldots, p_{n}}\left(\delta_{1}, \ldots, \delta_{n}\right)$.

From now on, we will consider that $p_{1}=p_{2}=\cdots=p_{n}$. As we did for the spaces $C^{p_{1}, \ldots, p_{n}}$, we will prove analogue generic results in the space $A^{p}(\Omega)$, where $\Omega$ is a planar domain bounded by the disjoint Jordan curves $\gamma_{1}, \ldots, \gamma_{n}$. More specifically, we will define the following spaces.

Definition 5.7 Let $p \in\{0,1, \ldots\} \cup\{\infty\}$ and let $\Omega$ be a bounded domain in $\mathbb{C}$. A function $f$ belongs to the class $A^{p}(\Omega)$ if it is holomorphic on $\Omega$ and every derivative $f^{(j)}$ can be continuously extended on $\bar{\Omega}$ for every $j \in\{0,1, \ldots\}, j \leq p$. The space $A^{p}(\Omega)$ is endowed with the topology of uniform convergence on $\bar{\Omega}$ of every derivative $f^{(j)}$ for all $j \in\{0,1, \ldots\}, j \leq p$ and becomes a complete metric space.

Remark 5.8 In particular cases it is true that $A^{p}(\Omega)$ is included in $C^{p}(\partial \Omega)$ as a closed subset. We will not examine now under which more general sufficient conditions this remains true.

Remark 5.9 If $\Omega$ is a (possibly unbounded) open set in $\mathbb{C}$, then a holomorphic function $f$ belongs to the class $A^{p}(\Omega)$ if for every $j \in\{0,1,2, \ldots\}, j \leq p$ the derivative $f^{(j)}$ has a continuous extension from $\Omega$ to its closure $\bar{\Omega}$ in $\mathbb{C}$. The topology of $A^{p}(\Omega)$ is defined by the seminorms $\sup \left|f^{(l)}(z)\right|, l \in\{0,1,2, \ldots\}, l \leq p, n \in \mathbb{N}$.

$$
z \in \bar{\Omega},|z| \leq n
$$

Definition 5.10 Let $\Omega$ be a bounded domain in $\mathbb{C}$ defined by a finite number of disjoint Jordan curves and $z_{0} \in \partial \Omega$. A continuous function $f: \bar{\Omega} \rightarrow \mathbb{C}$ belongs to the class of non-holomorphically extendable at $z_{0}$ functions in the sense of Riemann surfaces if there do not exist open disks $D\left(z_{0}, r\right), r>0$ and $D\left(z_{1}, d\right), z_{1} \in D\left(z_{0}, r\right) \cap \Omega, d>0$ such that $D\left(z_{1}, d\right) \subseteq D\left(z_{0}, r\right) \cap \Omega$, and a holomorphic function $F: D\left(z_{0}, r\right) \rightarrow \mathbb{C}$ such that $\left.F\right|_{D\left(z_{1}, d\right)}=\left.f\right|_{D\left(z_{1}, d\right)}$.

Theorem 5.11 Let $p \in\{0,1, \ldots\} \cup\{\infty\}$ and $\Omega$ be a bounded domain in $\mathbb{C}$ defined by a finite number of disjoint Jordan curves. Let also $z_{0} \in \partial \Omega$. The class of nonholomorphically extendable at $z_{0}$ functions of $A^{p}(\Omega)$ in the sense of Riemann surfaces is a dense and $G_{\delta}$ subset of $A^{p}(\Omega)$. 
Proof Let $M>0, r>0, z_{1} \in D\left(z_{0}, r\right) \cap \Omega$, and $d>0$ such that $D\left(z_{1}, d\right) \subset$ $D\left(z_{0}, r\right) \cap \Omega$. Let also $A\left(r, z_{1}, d, M\right)$ be the set of $A^{p}(\Omega)$ functions $f$ for which there exists a holomorphic function $F$ on $D\left(z_{0}, r\right)$, such that $|F(z)| \leq M$ for every $z \in D\left(z_{0}, r\right)$ and $\left.F\right|_{D\left(z_{1}, d\right)}=\left.f\right|_{D\left(z_{1}, d\right)}$. We will first show that $A\left(r, z_{1}, d, M\right)$ is a closed subset of $A^{p}(\Omega)$ with empty interior.

Let $\left(f_{n}\right)_{n \geq 1}$ be a sequence in $A\left(r, z_{1}, d, M\right)$ converging in the topology of $A^{p}(\Omega)$ to a function $f$ of $A^{p}(\Omega)$. Then, there are holomorphic functions $F_{n}: D\left(z_{0}, r\right) \rightarrow \mathbb{C}$ bounded by $M$ such that $\left.F_{n}\right|_{D\left(z_{1}, d\right)}=\left.f_{n}\right|_{D\left(z_{1}, d\right)}$. By Montel's Theorem there is a subsequence $\left(F_{k_{n}}\right)$ of $\left(F_{n}\right)$ which converges uniformly on the compact subsets of $D\left(z_{0}, r\right)$ to a function $F$ which is holomorphic and bounded by $M$ on $D\left(z_{0}, r\right)$. Since $F_{k_{n}}$ converges to $f$ on $D\left(z_{1}, d\right)$ we have that $\left.F\right|_{D\left(z_{1}, d\right)}=\left.f\right|_{D\left(z_{1}, d\right)}$ and so $f \in A$. Therefore, $A\left(r, z_{1}, d, M\right)$ is a closed subset of $A^{p}(\Omega)$.

Following the strategy of Theorem 4.7, if $A$ has non-empty interior, then there exist $f \in A\left(r, z_{1}, d, M\right), l \in\{0,1,2, \ldots\}$ and $\epsilon>0$, such that

$$
B=\left\{g \in A^{p}(\Omega): \sup _{z \in \bar{\Omega}}\left|f^{(j)}(z)-g^{(j)}(z)\right|<\epsilon, 0 \leq j \leq l\right\} \subset A\left(r, z_{1}, d, M\right) .
$$

We choose $w \in D\left(z_{0}, r\right) \backslash \bar{\Omega}$ and $0<\delta$ small enough such that the function $h(z)=$ $f(z)+\frac{\delta}{2(z-w)}$ belongs to $B$. By definition, $h$ has a holomorphic and bounded extension $H$ on $D\left(z_{0}, r\right)$. However, there is a holomorphic function $F: D\left(z_{0}, r\right) \rightarrow$ $\mathbb{C}$ which coincides with $f$ on $D\left(z_{1}, d\right)$. By analytic continuation $H(z)=F(z)+$ $\frac{\delta}{2(z-w)}$ for every $z \in D\left(z_{0}, r\right) \backslash\left\{z_{0}\right\}$, since they are equal on $D\left(z_{1}, d\right)$. As a result, $H$ is not bounded on $D\left(z_{0}, r\right)$ which yields the desired contradiction. Thus $A\left(r, z_{1}, d, M\right)$ has empty interior.

Next, let us consider $B$ be the set of $(r, z, d, M)$, where $r=1 / n, d=1 / m$ for some $n, m \in\{1,2, \ldots\}$ for which there exists $z \in \mathbb{Q}+i \mathbb{Q}$ such that $D(z, d) \subset D\left(z_{0}, r\right) \cap \Omega$, and $M \in\{1,2, \ldots\}$. Consider an enumeration $\left(b_{n}\right)$ of $B$. Then the class of nowhere holomorphically extendable functions of $A^{p}(\Omega)$ coincides with the set

$$
\bigcap_{n=1}^{\infty}\left(A^{p}(\Omega) \backslash A\left(b_{n}\right)\right)
$$

Thus, according to Baire's Theorem, the class of non-holomorphically extendable at $z_{0}$ functions of $A^{p}(\Omega)$ is dense and $G_{\delta}$.

Definition 5.12 Let $\Omega$ be a bounded domain in $\mathbb{C}$ defined by a finite number of disjoint Jordan curves. A continuous function $f: \bar{\Omega} \rightarrow \mathbb{C}$ belongs to the class of nowhere holomorphically extendable functions in the sense of Riemann surfaces if for every $z_{0} \in \partial \Omega f$ belongs to the class of non-holomorphically extendable at $z_{0}$ functions in the sense of Riemann surfaces.

Theorem 5.13 Let $p \in\{0,1, \ldots\} \cup\{\infty\}$ and let $\Omega$ be a bounded domain in $\mathbb{C}$ defined by a finite number of disjoint Jordan curves. The class of nowhere holomorphically 
extendable functions of $A^{p}(\Omega)$ in the sense of Riemann surfaces is a dense and $G_{\delta}$ subset of $A^{p}(\Omega)$.

Proof Let $z_{l}, l=1, \ldots$ be a dense sequence of $\partial \Omega$. The class $A\left(z_{l}\right)$ of nonholomorphically extendable at $z_{l}$ functions of $A^{p}(\Omega)$ in the sense of Riemann surface is a dense and $G_{\delta}$ subset of $A^{p}(\Omega)$ from Theorem 5.11. Notice that the set $\bigcap_{l=1}^{\infty} A\left(z_{l}\right)$ coincides with the class of nowhere holomorphically extendable functions of $A^{p}(\Omega)$ in the sense of Riemann surfaces and from Baire's Theorem is a dense and $G_{\delta}$ subset of $A^{p}(\Omega)$.

Remark 5.14 In [9] it has been also proved that the class of nowhere holomorphically extendable functions of $A^{\infty}(\Omega)$ in the sense of Riemann surfaces is a dense and $G_{\delta}$ subset of $A^{\infty}(\Omega)$. The method in [9] comes from the theory of Universal Taylor Series and is different from the method in the present paper.

Now we will examine a different kind of extendability.

Definition 5.15 Let $\Omega$ be a bounded domain in $\mathbb{C}$ defined by a finite number of disjoint Jordan curves $\gamma_{1}, \ldots, \gamma_{n}$. Let also $z_{0} \in \partial \Omega$. A continuous function $f: \bar{\Omega} \rightarrow \mathbb{C}$ belongs to the class of non-holomorphically extendable at $z_{0}$ functions if there exist no pair of an open disk $D\left(z_{0}, r\right), r>0$ and a holomorphic function $F: D\left(z_{0}, r\right) \rightarrow \mathbb{C}$ such that $F(z)=f(z)$ for every $z \in D\left(z_{0}, r\right) \cap \partial \Omega$. Otherwise we will say that $f$ is holomorphically extendable at $z_{0}$.

Remark 5.16 If $\gamma_{i}: I_{i} \rightarrow \mathbb{C}, I_{i}=\left[a_{i}, b_{i}\right], a_{i}<b_{i}$ is continuous and $\gamma_{i}(x)=\gamma_{i}(y)$ for $x, y \in\left[a_{i}, b_{i}\right]$ if and only if $x=y$ or $x, y \in\left\{a_{i}, b_{i}\right\}$, then we observe that a function $f$ belongs to the class of non-holomorphically extendable at $z_{0}=\gamma_{i}\left(t_{0}\right), t_{0} \in I_{i}^{\circ}$ of Definition 5.15 if and only if there are no open disk $D\left(z_{0}, r\right), r>0$, and $\eta>0$ and a holomorphic function $F: D\left(z_{0}, r\right) \rightarrow \mathbb{C}$, such that $\gamma_{i}\left(\left(t_{0}-\eta, t_{0}+\eta\right) \cap I_{i}\right) \subset D\left(z_{0}, r\right)$ and $F\left(\gamma_{i}(t)\right)=f\left(\gamma_{i}(t)\right)$ for all $t \in\left(t_{0}-\eta, t_{0}+\eta\right) \cap I_{i}$. This holds true because of the following observations:

1. For some constant $\eta>0$, we can find $r>0$ such that $D\left(z_{0}, r\right) \cap \gamma_{i}^{*} \subseteq \gamma_{i}\left(\left(t_{0}-\right.\right.$ $\left.\left.\eta, t_{0}+\eta\right) \cap I_{i}\right)$. This follows from the fact that the disjoint compact sets $\gamma_{i}\left[I_{i} \backslash\right.$ $\left.\left(t_{0}-\eta, t_{0}+\eta\right)\right]$ and $\left\{z_{0}\right\}$ have a strictly positive distance.

2. For some constant $r>0$ we can find $\eta>0$ such that $\gamma_{i}\left(\left(t_{0}-\eta, t_{0}+\eta\right) \cap I_{i}\right) \subset$ $D\left(z_{0}, r\right)$, because of the continuity of the map $\gamma_{i}$.

The above remark remains valid for $t_{0}=a_{i}$ or $t_{0}=b_{i}$, because a Jordan curve gamma can also be parametrized on $\left[a_{i}+\varepsilon, b_{i}+\varepsilon\right]$.

Theorem 5.17 Let $p \in\{0,1, \ldots\} \cup\{\infty\}$ and $\Omega$ be a bounded domain in $\mathbb{C}$ defined by a finite number of disjoint Jordan curves. Let also $z_{0} \in \partial \Omega$. The class of nonholomorphically extendable at $z_{0}$ functions of $A^{p}(\Omega)$ is a dense and $G_{\delta}$ subset of $A^{p}(\Omega)$.

Proof Let $M>0, r>0$, and $\mathcal{A}(r, M)=A\left(p, \Omega, z_{0}, r, M\right)$ be the class of functions $f \in A^{p}(\Omega)$ for which there exist a holomorphic function $F: D\left(z_{0}, r\right) \rightarrow \mathbb{C}$ such that $\left.F\right|_{D\left(z_{0}, r\right) \cap \partial \Omega}=\left.f\right|_{D\left(z_{0}, r\right) \cap \partial \Omega}$ and $|F(z)| \leq M$ for $z \in D\left(z_{0}, r\right)$. 
Similar to the proof of Lemma $4.1, \mathcal{A}(r, M)$ is a closed subset of $A^{p}(\Omega)$.

If $\mathcal{A}(r, M)$ does not have empty interior, then there exist a function $f$ in the interior of $\mathcal{A}(r, M)$, a number $b \in\{0,1,2, \ldots\}$, and $\delta>0$ such that

$$
\left\{g \in A^{p}(\Omega): \sup _{z \in \bar{\Omega}}\left|f^{(j)}(z)-g^{(j)}(z)\right|<\delta, 0 \leq j \leq b\right\} \subset \mathcal{A}(r, M) .
$$

Then similar to the proof of Theorem 4.7 choosing $w \in D\left(z_{0}, r\right) \backslash \bar{\Omega}$ and $a$ small enough, we are led to a contradiction. Thus $\mathcal{A}(r, M)$ has empty interior.

Notice that the set

$$
\bigcap_{M=1}^{\infty} \bigcap_{n=1}^{\infty}\left(A^{p}(\Omega) \backslash \mathcal{A}(1 / n, M)\right)
$$

coincides with the class of non-holomorphically extendable at $z_{0}$ functions of $A^{p}(\Omega)$ and Baire's Theorem implies that this set is a dense and $G_{\delta}$ subset of $A^{p}(\Omega)$.

Definition 5.18 Let $\Omega$ be a bounded domain in $\mathbb{C}$ defined by a finite number of disjoint Jordan curves. A continuous function $f: \bar{\Omega} \rightarrow \mathbb{C}$ belongs to the class of nowhere holomorphically extendable functions if for every $z_{0} \in \partial \Omega, f$ belongs to the class of non-holomorphically extendable at $z_{0}$ functions.

Theorem 5.19 Let $p \in\{0,1, \ldots\} \cup\{\infty\}$ and let $\Omega$ be a bounded domain in $\mathbb{C}$ defined by a finite number of disjoint Jordan curves. The class of nowhere holomorphically extendable functions of $A^{p}(\Omega)$ is a dense and $G_{\delta}$ subset of $A^{p}(\Omega)$.

Proof The proof is similar to the proof of Theorem 4.9, taking into account the statement of Theorem 5.17.

Remark 5.20 If the continuous analytic capacity of the boundary of $\Omega$ is zero, then Definition 5.10 implies Definition 5.15.

Now as in Sect. 4, we will associate the phenomenon of non-extendability with that of real analyticity on the spaces $A^{p}(\Omega)$.

Definition 5.21 Let $n \in\{1,2, \ldots\}$ and let $\Omega$ be a bounded domain in $\mathbb{C}$ defined by disjoint Jordan curves $\gamma_{1}, \ldots, \gamma_{n}$. A function $f: \bar{\Omega} \rightarrow \mathbb{C}$ is real analytic at $\left(t_{0}, \gamma_{i}\left(t_{0}\right)\right)$, $\gamma_{i}\left(t_{0}\right) \in \gamma_{i}^{*}, i \in\{1,2, \ldots, n\}$ if $\left.f\right|_{\gamma_{i}}$ is real analytic at $\left(t_{0}, \gamma_{i}\left(t_{0}\right)\right)$.

At this point we observe that if $\Omega$ is a bounded domain in $\mathbb{C}$ defined by disjoint Jordan curves $\gamma_{1}, \ldots, \gamma_{n}$ and $f \in A^{p}(\Omega)$, then the analogous of Proposition 2.9 under the above assumptions holds true, since nothing essential changes in its proof. So, we have the following proposition:

Proposition 5.22 Let $p \in\{0,1, \ldots\} \cup\{\infty\}, n \in\{0,1, \ldots\}$ and let $\Omega$ be a bounded domain in $\mathbb{C}$ defined by disjoint analytic Jordan curves $\gamma_{1}, \ldots, \gamma_{n}$. A continuous function $f: \bar{\Omega} \rightarrow \mathbb{C}$ is real analytic at $\left(t_{0}, \gamma_{i}\left(t_{0}\right)\right), \gamma_{i}\left(t_{0}\right) \in \gamma_{i}^{*}, i \in\{1,2, \ldots, n\}$ if and only if is holomorphically extendable at $\gamma_{i}\left(t_{0}\right)$. 
Definition 5.23 Let $p \in\{0,1, \ldots\} \cup\{\infty\}, n \in\{0,1, \ldots\}$ and let $\Omega$ be a bounded domain in $\mathbb{C}$ defined by disjoint Jordan curves $\gamma_{1}, \ldots, \gamma_{n}$. A function $f \in A^{p}(\Omega)$ is nowhere real analytic if there exist no $i \in\{1,2, \ldots, n\}$ and $\gamma_{i}\left(t_{0}\right) \in \partial \Omega$ such that $f$ is real analytic at $\left(t_{0}, \gamma_{i}\left(t_{0}\right)\right)$.

Now combining Proposition 5.22 with Theorem 5.19, we obtain the following theorem.

Theorem 5.24 Let $p \in\{0,1, \ldots\} \cup\{\infty\}$ and let $\Omega$ be a bounded domain in $\mathbb{C}$ defined by a finite number of disjoint analytic Jordan curves. The class of nowhere real analytic functions of $A^{p}(\Omega)$ is a dense and $G_{\delta}$ subset of $A^{p}(\Omega)$.

Remark 5.25 We recall that for an analytic Jordan curve $\gamma$ defined on $[0,1]$ there exist $0<r<1<R$ and a holomorphic injective function $\Phi: D(0, r, R) \rightarrow \mathbb{C}$, such that $\gamma(t)=\Phi\left(e^{i t}\right)$, where $D(0, r, R)=\{z \in \mathbb{C}: r<|z|<R\}$. This yields a natural parametrization of the curve $\gamma^{*}$; the parameter $t$ is called a conformal parameter for the curve $\gamma^{*}$. Theorem 5.24 holds if each of the Jordan curves $\gamma_{1}, \ldots, \gamma_{n}$ is parametrized by such a conformal parameter $t$. Naturally one asks if the same result holds for other parametrizations; for instance, does Theorem 5.24 remain true if each $\gamma_{1}, \ldots, \gamma_{n}$ is parametrized by arc length? This was the motivation of [10], where it is proved that arc length is a global conformal parameter for any analytic curve. Thus Theorem 5.24 remains also true if arc length is used as a parametrization for each analytic curve $\gamma_{i}$.

\section{One-Sided Extendability}

In this section, we consider one-sided extensions from a locally injective curve $\gamma$. For instance, if $\gamma^{*}$ is homeomorphic to $[0,1]$, one can find an open disk $D$ and an open arc $J$ of $\gamma^{*}$ separating $D$ to two components $D^{+}$and $D^{-}$. Those are Jordan domains containing in their boundaries a subarc $J$ of $\gamma$. We will show that generically in $C^{k}(\gamma)$ every function $h$ cannot be extended to a function $F: \Omega \cup J \rightarrow \mathbb{C}$ continuous on $\Omega \cup J$ and holomorphic on $\Omega$, where $\Omega=D^{+}$or $\Omega=D^{-}$. That is, the one-sided extendability is a rare phenomenon in $C^{k}(\gamma)$, provided that $\gamma$ is of class at least $C^{k}$. In order to prove this fact we need the following lemmas, which are well known in algebraic topology. The interested reader can find their proofs in [1].

Lemma 6.1 Let $\delta:[0,1] \rightarrow \mathbb{C}$ be a continuous and injective curve. Then the interior of $\delta^{*}$ in $\mathbb{C}$ is void.

Proposition 6.2 Let $\gamma: I \rightarrow \mathbb{C}$ be a continuous and locally injective curve. Then the interior of $\gamma^{*}$ in $\mathbb{C}$ is void.

Proposition 6.2 implies the following.

Corollary 6.3 Let $\gamma: I \rightarrow \mathbb{C}$ be a continuous and locally injective curve and $\Omega$ be a Jordan domain whose boundary contains an arc $\gamma\left(\left[t_{1}, t_{2}\right]\right), t_{1}<t_{2}, t_{1}, t_{2} \in I$ of $\gamma^{*}$. Then the set $\Omega \backslash \gamma^{*}$ is non-empty. 
Let $\gamma: I \rightarrow \mathbb{C}$ be a continuous and locally injective curve defined on the interval $I \subset \mathbb{R}$. Naturally, one asks if a Jordan domain as in Corollary 6.3 exists. Our goal is to construct denumerably many such Jordan domains, so that every $\Omega$, as in Corollary 6.3, contains one of these domains and then use Baire's Category Theorem.

Let $t_{0}$ in the interior $I^{\circ}$ of $I$ and $\Omega$ be a Jordan domain whose boundary contains an $\operatorname{arc} \gamma\left(\left[t_{1}, t_{2}\right]\right), t_{1}<t_{0}<t_{2}$. Let us assume that $t_{1}, t_{2}$ are rational numbers. Pick $s_{1}, s_{2} \in$ $\left(t_{1}, t_{2}\right) \cap \mathbb{Q}$ such that $s_{1}<t_{0}<s_{2}$. By definition, the point $\gamma\left(s_{1}\right)$ does not belong to the compact set $\Omega_{1}:=\partial \Omega \backslash \gamma\left(t_{1}, t_{0}\right)$. As a result their distance $\delta:=\operatorname{dist}\left(\gamma\left(s_{1}\right), \Omega_{1}\right)$ is positive. From Proposition 6.2 there exists $P \in(\mathbb{Q}+i \mathbb{Q}) \cap D\left(\gamma\left(s_{1}\right), \delta / 2\right) \cap \Omega$ with $P \notin \gamma\left(\left[t_{1}, t_{0}\right]\right)$. Notice that there is $r \in\left(t_{1}, t_{0}\right)$ such that $|\gamma(r)-P|=a$, where $a:=\operatorname{dist}\left(P, \gamma\left(\left[t_{1}, t_{0}\right]\right)\right) \leq\left|P-\gamma\left(s_{1}\right)\right|<\delta / 2$, because for every $z \in \Omega_{1}$ we have

$$
|z-P| \geq\left|z-\gamma\left(s_{1}\right)\right|-\left|\gamma\left(s_{1}\right)-P\right|>\delta-\delta / 2=\delta / 2 .
$$

By definition, the segment $[P, \gamma(r)]$ intersects $\partial \Omega$ only at $\gamma(r)$. Similarly we pick $Q \in(\mathbb{Q}+i \mathbb{Q}) \cap \Omega$ and $\widetilde{r} \in\left(t_{0}, t_{2}\right)$, such that the segment $[Q, \gamma(\widetilde{r})]$ intersects $\partial \Omega$ only at $\gamma(\widetilde{r})$.

We distinguish two cases according to whether the segments $[P, \gamma(r)],[Q, \gamma(\widetilde{r})]$ intersect or not. If the segments $[P, \gamma(r)],[Q, \gamma(\widetilde{r})]$ intersect at a point $w$, then the union of the segments $[w, \gamma(r)],[w, \gamma(\widetilde{r})]$, and $\gamma[\widetilde{r}, r]$ is the image of a Jordan curve, the interior of which is the desired Jordan domain. If the segments $[P, \gamma(r)]$, $[Q, \gamma(\widetilde{r})]$ do not intersect, then we consider a simple polygonal line (that is without self-intersections) in $\Omega$, which connects $P, Q$, the vertices of which belong to $\mathbb{Q}+i \mathbb{Q}$. This is possible, since $\Omega$ is a domain [11]. The union of the aforementioned polygonal line with the segments $[P, \gamma(r)]$ and $[Q, \gamma(\widetilde{r})]$ has possibly self-intersections. For this reason, we consider one of the connected components of this simple polygonal line minus the segments $[P, \gamma(r)],[Q, \gamma(\widetilde{r})]$ with the property that the closure of this connected component is a simple polygonal line connecting two points $z_{1} \in[P, \gamma(r)]$, $z_{2} \in[Q, \gamma(\widetilde{r})]$. Then the union of the last simple polygonal line with the segments $\left[z_{1}, \gamma(r)\right],\left[z_{2}, \gamma(\widetilde{r})\right]$ and the arc $\gamma[\widetilde{r}, r]$ of $\gamma^{*}$ is the image of a Jordan curve, the interior of which is the desired Jordan domains. We notice that $t_{0} \in[\widetilde{r}, r]$ and that the constructed Jordan domains are denumerably many.

Proposition 6.4 Let $\gamma: I \rightarrow \mathbb{C}$ be a locally injective map of class $C^{l}(I), l \in$ $\{0,1,2, \ldots\} \cup\{\infty\}$, and $0<M<\infty$. Let also $\Omega$ be a Jordan domain whose boundary contains an arc $\gamma\left(\left[t_{1}, t_{2}\right]\right), t_{1}<t_{2}, t_{1}, t_{2} \in I$ of $\gamma^{*}$ and $k \in\{0,1,2, \ldots\} \cup\{\infty\}$, $k \leq l$. The set of functions $f \in C^{k}(\gamma)$ for which there exists a continuous function $F: \Omega \cup \gamma\left(\left(t_{1}, t_{2}\right)\right),\|F\|_{\infty} \leq M$, such that $F$ is holomorphic on $\Omega$ and $\left.F\right|_{\gamma\left(\left(t_{1}, t_{2}\right)\right)}=$ $\left.f\right|_{\gamma\left(\left(t_{1}, t_{2}\right)\right)}$, is a closed subset of $C^{k}(\gamma)$ with empty interior.

Proof Let $\Psi$ be a homeomorphism of $D \cup J \subset \mathbb{C}$ on $\Omega \cup \gamma\left(t_{1}, t_{2}\right)$, which is also holomorphic on $\Omega$, where $J=\left\{e^{i t}: 0<t<1\right\}$. This is possible because of the Caratheodory-Osgood Theorem. Let also $A(k, \Omega, M)$ be the set of functions $f \in$ $C^{k}(\gamma)$ for which there exists a continuous function $F: \Omega \cup \gamma\left(\left(t_{1}, t_{2}\right)\right) \rightarrow \mathbb{C},\|F\|_{\infty} \leq$ $M$, such that $F$ is holomorphic on $\Omega$ and $\left.F\right|_{\gamma\left(\left(t_{1}, t_{2}\right)\right)}=\left.f\right|_{\gamma\left(\left(t_{1}, t_{2}\right)\right)}$.

First, we will prove that $A(k, \Omega, M)$ is a closed subset of $C^{k}(\gamma)$. Let $\left(f_{n}\right)_{n \geq 1}$ be a sequence in $A(k, \Omega, M)$ converging in the topology of $C^{k}(\gamma)$ to a function $f \in C^{l}(\gamma)$. 
This implies that $f_{n}$ converges uniformly on the compact subsets of $\gamma^{*}$ to $f$. Then for $n=1,2, \ldots$ there exist continuous functions $F_{n}: \Omega \cup \gamma\left(\left(t_{1}, t_{2}\right)\right) \rightarrow \mathbb{C},\left\|F_{n}\right\|_{\infty} \leq M$, such that $F_{n}$ are holomorphic on $\Omega$ and $\left.F_{n}\right|_{\gamma\left(\left(t_{1}, t_{2}\right)\right)}=\left.f_{n}\right|_{\gamma\left(\left(t_{1}, t_{2}\right)\right)}$. If $G_{n}=F_{n} \circ \Psi$, $g_{n}=f_{n} \circ \Psi$ and $g=f \circ \Psi$ for $n=0,1,2, \ldots$, it follows that $g_{n}$ converges uniformly on the compact subsets of $J$ to $g$. Also, the functions $G_{n}$ are holomorphic and bounded by $M$ on $D$. By Montel's Theorem, there exists a subsequence of $\left(G_{n}\right)$, $\left(G_{k_{n}}\right)$ which converges uniformly on the compact subsets of $D$ to a function $G$ which is holomorphic and bounded by $M$ on $D$. Without loss of generality, we assume that $\left(G_{n}\right)=\left(G_{k_{n}}\right)$. Now it is sufficient to prove that for any circular sector $K$, which has boundary $\left[0, e^{i a}\right] \cup\left[0, e^{i b}\right] \cup\left\{e^{i t}: a \leq t \leq b\right\}, 0<a<b<1$, the sequence $\left(G_{n}\right)$ converges uniformly on $K$, because then the limit of $\left(G_{n}\right)$, which is equal to $g$ at the arc $J$ and equal to $G$ on the remaining part of the circular sector $K$, will be a continuous function. In order to do so we will prove that $\left(G_{n}\right)$ forms a uniformly Cauchy sequence on $K$. Since each $G_{n}$ is a bounded holomorphic function on $D$, we know that for every $n$ the radial limits of $G_{n}$ exist almost everywhere on the unit circle and so we can consider the respective functions $g_{n}$ defined almost everywhere on the unit circle which are extensions of the previous $g_{n}$. These $g_{n}$ are also bounded by $M$. Let $\varepsilon>0$. Using the identity $G_{n}\left(r e^{i \theta}\right)=\frac{1}{2 \pi} \int_{-\pi}^{\pi} P_{r}(t) g_{n}(\theta-t) d t$, where $P_{r}$ is the Poisson kernel, we obtain

$$
\left|G_{n}\left(r e^{i \theta}\right)-G_{m}\left(r e^{i \theta}\right)\right| \leq \frac{1}{2 \pi} \int_{-\pi}^{\pi} P_{r}(t)\left|g_{n}(\theta-t)-g_{m}(\theta-t)\right| d t .
$$

Our strategy is to split the integral into two parts. We choose $0<\delta<\min \{1-b, a\}$ and pick $r_{0} \in(0,1)$ such that $\sup _{\delta \leq|t| \leq \pi} P_{r}(t)<\frac{\varepsilon}{8 M}$ for every $r \in\left[r_{0}, 1\right)$. Then $\left(G_{n}\right)$ is a uniformly Cauchy sequence on $K \cap\left\{z \in \mathbb{C}:|z| \leq r_{0}\right\}$, and thus there exists $n_{1}$ such that for every $n, m \geq n_{1}$,

$$
\sup _{z \in K \cap\left\{z \in \mathbb{C}:|z| \leq r_{0}\right\}}\left|G_{n}(z)-G_{m}(z)\right|<\frac{\varepsilon}{2} .
$$

In addition, as $g_{n}$ converges uniformly to $g$ on $J$ there exists $n_{2}$, such that for every $n, m \geq n_{2}, \sup _{z \in J}\left|g_{n}(z)-g_{m}(z)\right|<\frac{\varepsilon}{4}$. Consequently, for $n, m \geq \max \left\{n_{1}, n_{2}\right\}, \theta \in$ $[a, b]$, and $r \in\left(r_{0}, 1\right)$, we obtain

$$
\frac{1}{2 \pi} \int_{-\delta}^{\delta} P_{r}(t)\left|g_{n}(\theta-t)-g_{m}(\theta-t)\right| d t \leq \frac{\varepsilon}{4} \frac{1}{2 \pi} \int_{-\delta}^{\delta} P_{r}(t) d t \leq \frac{\varepsilon}{4}
$$

and

$$
\frac{1}{2 \pi} \int_{\delta \leq|t| \leq \pi} P_{r}(t)\left|g_{n}(\theta-t)-g_{m}(\theta-t)\right| d t \leq \frac{\sup _{\delta \leq|t| \leq \pi} P_{r}(t)}{2 \pi} \int_{\delta \leq|t| \leq \pi} 2 M d t \leq \frac{\varepsilon}{4} .
$$


Therefore $\left|G_{n}\left(r e^{i \theta}\right)-G_{m}\left(r e^{i \theta}\right)\right| \leq \frac{\varepsilon}{2}$ and by the continuity of the functions $G_{n}$ on $D \cup J$, making $r \rightarrow 1^{-}$we find that $\left|G_{n}\left(e^{i \theta}\right)-G_{m}\left(e^{i \theta}\right)\right| \leq \frac{\varepsilon}{2}$ for every $\theta \in[a, b]$. It follows immediately that $\left(G_{n}\right)$ is a uniformly Cauchy sequence on the circular sector $K$ and thus the set $A(k, \Omega, M)$ is a closed subset of $C^{k}(\gamma)$.

If $A(k, \Omega, M)$ does not have empty interior, then there exists a function $f$ in the interior of $A(k, \Omega, M)$, a compact set $L \subset I$ and $\delta>0$ such that

$$
\left\{g \in C^{k}(\gamma): \sup _{t \in L}\left|(f \circ \gamma)^{(j)}(t)-(g \circ \gamma)^{(j)}(t)\right|<\delta, 0 \leq j \leq b\right\} \subset A(k, \Omega, M) .
$$

From Corollary 6.3, we can find $w \in \Omega \backslash \gamma^{*}$. We choose $0<a<\delta \min \left\{\inf _{t \in K} \mid \gamma(t)-\right.$ $\left.w\left|, \ldots, \frac{1}{b !} \inf _{t \in K}\right| \gamma(t)-\left.w\right|^{b+1}\right\}$. The function $h(\gamma(t))=f(\gamma(t))+\frac{a}{2(\gamma(t)-w)}, t \in I$ belongs to $A(k, \Omega, M)$ and therefore has a continuous and bounded extension $H$ on $\Omega \cup \gamma\left(\left(t_{1}, t_{2}\right)\right)$ with $\left.H\right|_{\gamma\left(\left(t_{1}, t_{2}\right)\right)}=\left.h\right|_{\gamma\left(\left(t_{1}, t_{2}\right)\right)}$ which is holomorphic on $\Omega$. Then the function $H \circ \Psi$ is continuous and bounded on $D \cup J$ and holomorphic on $D$. It is easy to see that $H(\Psi(z))=F(\Psi(z))+\frac{a}{2(\Psi(z)-w)}$ for $z \in D \backslash\left\{\Psi^{-1}(w)\right\}$. Indeed, let $\Phi(z)=H(\Psi(z))-F(\Psi(z))-\frac{a}{2(\Psi(z)-w)}$. Then $\left.\Phi\right|_{J}=0$ and by Schwarz Reflection Principle $\Phi$ is extended holomorphically on

$$
(D \cup J \cup(\mathbb{C} \backslash \bar{D})) \backslash\left\{\Psi^{-1}(w), \frac{1}{\overline{\Psi^{-1}(w)}}\right\} .
$$

Therefore because $\Phi=0$ on $J$, by analytic continuation, $H(\Psi(z))-F(\Psi(z))-$ $\frac{a}{2(\Psi(z)-w)}=0$ on $(D \cup J) \backslash\left\{\Psi^{-1}(w)\right\}$. As a result $H \circ \Psi$ is not bounded on $D$ which is absurd. Thus $A(k, \Omega, M)$ has empty interior.

Definition 6.5 Let $\gamma: I \rightarrow \mathbb{C}$ be a locally injective map and $t_{0} \in I^{\circ}$, where $I^{\circ}$ is the interior of $I$ in $\mathbb{R}$. A function $f: \gamma^{*} \rightarrow \mathbb{C}$ is non- one-sided holomorphically extendable at $\left(t_{0}, \gamma\left(t_{0}\right)\right)$ if there is no pair of a Jordan domain $\Omega$, such that $\partial \Omega$ contains an $\operatorname{arc}$ of $\gamma^{*}, \gamma\left(\left[t_{1}, t_{2}\right]\right), t_{1}<t_{0}<t_{2}, t_{1}, t_{2} \in I$ and a continuous function $F$ : $\Omega \cup \gamma\left(\left(t_{1}, t_{2}\right)\right)$, which is holomorphic on $\Omega$ and $\left.F\right|_{\gamma\left(\left(t_{1}, t_{2}\right)\right)}=\left.f\right|_{\gamma\left(\left(t_{1}, t_{2}\right)\right)}$.

Theorem 6.6 Let $\gamma: I \rightarrow \mathbb{C}$ be a locally injective map of class $C^{l}(I), l \in$ $\{0,1,2, \ldots\} \cup\{\infty\}$, and $k \in\{0,1,2, \ldots\} \cup\{\infty\}, k \leq l$. Consider $t_{0} \in I^{\circ}$, where $I^{\circ}$ is the interior of $I$ in $\mathbb{R}$. The class of non-one-sided holomorphically extendable at $\left(t_{0}, \gamma\left(t_{0}\right)\right)$ functions of $C^{k}(\gamma)$ is a dense and $G_{\delta}$ subset of $C^{k}(\gamma)$.

Proof Let $\Omega$ be a Jordan domain whose boundary contains an arc $\gamma\left(\left[t_{1}, t_{2}\right]\right)$, of $\gamma^{*}$, where $t_{1}<t_{0}<t_{2}$. $A(k, \Omega, M)$ denotes the set of functions $f \in C^{k}(\gamma)$ for which there is a continuous function $F: \Omega \cup \gamma\left(\left(t_{1}, t_{2}\right)\right)$, which is holomorphic on $\Omega$, bounded by $M$ and $\left.F\right|_{\gamma\left(\left(t_{1}, t_{2}\right)\right)}=\left.f\right|_{\gamma\left(\left(t_{1}, t_{2}\right)\right)}$. Let $G_{n}, n \geq 1$ be the denumerably many Jordan domains constructed above. 
From Proposition 6.4, the sets $A\left(k, G_{n}, M\right)$ are closed subsets of $C^{k}(\gamma)$ with empty interior. We will prove that the class of non-one-sided holomorphically extendable at $\left(t_{0}, \gamma\left(t_{0}\right)\right)$ functions of $C^{k}(\gamma)$ coincides with the set

$$
\bigcap_{n=1}^{\infty} \bigcap_{M=1}^{\infty}\left(C^{k}(\gamma) \backslash A\left(k, G_{n}, M\right)\right),
$$

and thus Baire's Theorem will imply that the above set is a dense and $G_{\delta}$ subset of $C^{k}(\gamma)$.

Obviously, the set

$$
\bigcap_{n=1}^{\infty} \bigcap_{M=1}^{\infty}\left(C^{k}(\gamma) \backslash A\left(k, G_{n}, M\right)\right)
$$

contains the class of non-one-sided holomorphically extendable at $\left(t_{0}, \gamma\left(t_{0}\right)\right)$ functions of $C^{k}(\gamma)$. Conversely, let $\Omega$ be a Jordan domain whose boundary contains an arc $\gamma\left(\left[t_{1}, t_{2}\right]\right), t_{1}<t_{0}<t_{2}, t_{1}, t_{2} \in I$. Let also $f \in C^{k}(\gamma)$, for which there is a continuous function $F: \Omega \cup \gamma\left(\left(t_{1}, t_{2}\right)\right)$, which is holomorphic on $\Omega$ and $\left.F\right|_{\gamma\left(\left(t_{1}, t_{2}\right)\right)}=\left.f\right|_{\gamma\left(\left(t_{1}, t_{2}\right)\right)}$. From the construction of the aforementioned Jordan domains $G_{n}$, we can find a Jordan domain $G_{n_{0}}$ such that $\overline{G_{n_{0}}}$ is contained in $\bar{\Omega}$. It easily follows that $\left.F\right|_{G_{n_{0}}}$ is bounded by some number $M=1,2,3, \ldots$ and thus $f$ belongs to $A\left(k, G_{n_{0}}, M\right)$. Therefore the class of non- one-sided holomorphically extendable at $\left(t_{0}, \gamma\left(t_{0}\right)\right)$ functions of $C^{k}(\gamma)$ is a subset of the set

$$
\bigcap_{n=1}^{\infty} \bigcap_{M=1}^{\infty}\left(C^{k}(\gamma) \backslash A\left(k, G_{n}, M\right)\right),
$$

which combined with the above completes the proof.

Definition 6.7 Let $\gamma: I \rightarrow \mathbb{C}$ be a locally injective map on the interval $I \subset \mathbb{R}$. A function $f: \gamma^{*} \rightarrow \mathbb{C}$ is nowhere one-sided holomorphically extendable if there is no pair of a Jordan domain $\Omega$, such that $\partial \Omega$ contains an arc of $\gamma^{*}, \gamma\left(\left[t_{1}, t_{2}\right]\right), t_{1}<t_{2}$, $t_{1}, t_{2} \in I$ and a continuous function $F: \Omega \cup \gamma\left(\left(t_{1}, t_{2}\right)\right)$, which is holomorphic on $\Omega$ and $\left.F\right|_{\gamma\left(\left(t_{1}, t_{2}\right)\right)}=\left.f\right|_{\gamma\left(\left(t_{1}, t_{2}\right)\right)}$.

Theorem 6.8 Let $\gamma: I \rightarrow \mathbb{C}$ be a locally injective map of class $C^{l}, l \in\{0,1,2, \ldots\} \cup$ $\{\infty\}$ on the interval $I \subset \mathbb{R}$ and $k \in\{0,1,2, \ldots\} \cup\{\infty\}, k \leq l$. The class of nowhere one-sided holomorphically extendable functions of $C^{k}(\gamma)$ is a dense and $G_{\delta}$ subset of $C^{k}(\gamma)$.

Proof Let $t_{n} \in I^{\circ}, n=1,2, \ldots$ be a sequence which is dense in $I$. Then the class of nowhere one-sided holomorphically extendable functions of $C^{k}(\gamma)$ coincides with the intersection of the classes of non-one-sided holomorphically extendable at $\left(t_{n}, \gamma\left(t_{n}\right)\right)$ functions of $C^{k}(\gamma)$, which is from Theorem 6.6 and Baire's Theorem a dense and $G_{\delta}$ subset of $C^{k}(\gamma)$. 
Let $\gamma_{i}: I_{i} \rightarrow \mathbb{C}$ be continuous and locally injective curves. As we did in Sect. 5, we will prove generic results for the spaces $C^{p_{1}, \ldots, p_{n}}\left(\gamma_{1}, \ldots, \gamma_{n}\right)$.

Definition 6.9 Let $n \in\{1,2, \ldots\}$ and $\gamma_{i}: I_{i} \rightarrow \mathbb{C}, i=1, \ldots, n$, be locally injective curves, where $I_{i}$ are intervals and $t_{0}$ a point of some $I_{i_{0}}$. A function $f$ on the disjoint union $\gamma_{1}^{*} \cup \cdots \cup \gamma_{n}^{*}, f(z)=f_{i}(z)$ for $z \in \gamma_{i}^{*}$, is non-one-sided holomorphically extendable at $\left(t_{0}, \gamma_{i_{0}}\left(t_{0}\right)\right)$ if the function $f_{i_{0}}$ defined on $\gamma_{i_{0}}^{*}$ is non-one-sided holomorphically extendable at $\left(t_{0}, \gamma_{i_{0}}\left(t_{0}\right)\right)$.

Theorem 6.10 Let $n \in\{1,2, \ldots\}$ and $p_{i}, q_{i} \in\{0,1, \ldots\} \cup\{\infty\}$ such that $p_{i} \leq q_{i}$ for every $i=1, \ldots, n$. Let also $\gamma_{i}: I_{i} \rightarrow \mathbb{C}$ be locally injective functions of class $C^{q_{i}}\left(I_{i}\right)$, where $I_{i}$ are intervals, and $t_{0}$ a point of some $I_{i_{0}}$. The class of non-one-sided holomorphically extendable at $\left(t_{0}, \gamma_{i_{0}}\left(t_{0}\right)\right)$ functions belonging to $C^{p_{1}, \ldots, p_{n}}\left(\gamma_{1}, \ldots, \gamma_{n}\right)$ is a dense and $G_{\delta}$ subset of $C^{p_{1}, \ldots, p_{n}}\left(\gamma_{1}, \ldots, \gamma_{n}\right)$.

Proof Similar to the proof of Theorem 5.4.

Definition 6.11 Let $n \in\{1,2, \ldots\}$ and $\gamma_{i}: I_{i} \rightarrow \mathbb{C}$ be locally injective curves, where $I_{i}$ are intervals. A function $f$ on the disjoint union $\gamma_{1}^{*} \cup \cdots \cup \gamma_{n}^{*}, f(z)=f_{i}(z)$ for $z \in \gamma_{i}^{*}$, is nowhere one-sided holomorphically extendable if the functions $f_{i}$ defined on $\gamma_{i}^{*}$ are nowhere one-sided holomorphically extendable functions.

The proof of the following theorem is again similar to the proof of Theorem 5.4.

Theorem 6.12 Let $n \in\{1,2, \ldots\}, p_{i}, q_{i} \in\{0,1, \ldots\} \cup\{\infty\}$ such that $p_{i} \leq q_{i}$ for $i=1, \ldots, n$. Let also $\gamma_{i}: I_{i} \rightarrow \mathbb{C}$ be locally injective functions of $C^{q_{i}}\left(I_{i}\right)$, where $I_{i}$ are intervals. The class of nowhere one-sided holomorphically extendable functions belonging to $C^{p_{1}, \ldots, p_{n}}\left(\gamma_{1}, \ldots, \gamma_{n}\right)$ is a dense and $G_{\delta}$ subset of $C^{p_{1}, \ldots, p_{n}}\left(\gamma_{1}, \ldots, \gamma_{n}\right)$.

Definition 6.13 Let $n \in\{1,2, \ldots\}$ and $\Omega$ be a bounded domain in $\mathbb{C}$ defined by disjoint periodic Jordan curves $\gamma_{i}: \mathbb{R} \rightarrow \mathbb{C}$. Let also $t_{0} \in \mathbb{R}$. A function $f: \bar{\Omega} \rightarrow \mathbb{C}$ is non-one-sided holomorphically extendable at $\left(t_{0}, \gamma_{i_{0}}\left(t_{0}\right)\right)$ outside $\Omega$ if there is no pair of a Jordan domain $G \subset \mathbb{C} \backslash \bar{\Omega}$, such that $\partial G$ contains a Jordan arc $\gamma_{i_{0}}\left(\left[t_{1}, t_{2}\right]\right)$ of $\gamma_{i_{0}}^{*}$ and a continuous function $F: \Omega \cup \gamma\left(\left(t_{1}, t_{2}\right)\right)$, which is holomorphic on $G$ and $\left.F\right|_{\gamma_{i_{0}}\left(\left(t_{1}, t_{2}\right)\right)}=\left.f\right|_{\gamma_{i_{0}}\left(\left(t_{1}, t_{2}\right)\right)}$.

The following theorem is a simple combination of proofs similar to those of Proposition 6.4 and Theorem 6.6.

Theorem 6.14 Let $p \in\{0,1, \ldots\} \cup\{\infty\}, n \in\{1,2, \ldots\}$ and $\Omega$ be a bounded domain in $\mathbb{C}$ defined by disjoint periodic Jordan curves $\gamma_{i}: \mathbb{R} \rightarrow \mathbb{C}$. Let also $t_{0} \in \mathbb{R}$. The class of non-one-sided holomorphically extendable at $\left(t_{0}, \gamma_{i_{0}}\left(t_{0}\right)\right)$ outside $\Omega$ functions of $A^{p}(\Omega)$ is a dense and $G_{\delta}$ subset of $A^{p}(\Omega)$.

Definition 6.15 Let $n \in\{1,2, \ldots\}$ and $\Omega$ be a bounded domain in $\mathbb{C}$ defined by disjoint periodic Jordan curves $\gamma_{i}: \mathbb{R} \rightarrow \mathbb{C}$. A function $f: \bar{\Omega} \rightarrow \mathbb{C}$ is nowhere onesided holomorphically extendable outside $\Omega$ if $f$ is non-one-sided holomorphically extendable at $\left(t_{0}, \gamma_{i_{0}}\left(t_{0}\right)\right)$ outside $\Omega$ for every $t_{0} \in \mathbb{R}$ and $i_{0}=1,2, \ldots, n$. 
Combining Theorem 6.14 for a dense in $\mathbb{R}$ sequence $t_{n}$ and Baire's Theorem, we obtain the following:

Theorem 6.16 Let $p \in\{0,1, \ldots\} \cup\{\infty\}$ and let $\Omega$ be a bounded domain in $\mathbb{C}$ defined by a finite number of disjoint Jordan curves. The class of nowhere one-sided holomorphically extendable functions outside of $\Omega$ is a dense and $G_{\delta}$ subset of $A^{p}(\Omega)$.

\section{Removability of Singularities in the Spaces $A^{p}$ and the $p$-Continuous Analytic Capacities: A Dichotomy Result}

Let $\Omega$ denote an open and bounded subset of $\mathbb{C}$ and $L$ be a compact subset of $\Omega$. Consider the open set $G=\Omega \backslash L, p \in\{0,1, \ldots\} \cup\{\infty\}$ and $f_{0} \in A^{p}(G)$. Then there are two cases:

(i) Either there is a function $F_{0} \in A^{p}(\Omega)$, such that $\left.F_{0}\right|_{G}=f_{0}$

(ii) or there exists no $F_{0} \in A^{p}(\Omega)$, such that $\left.F_{0}\right|_{G}=f_{0}$.

Theorem 7.1 Let $p \in\{0,1, \ldots\} \cup\{\infty\}, \Omega$ be an open and bounded subset of $\mathbb{C}$ and $L$ be a compact subset of $\Omega$. Let also $G=\Omega \backslash L$. Suppose that there is a function $f_{0} \in A^{p}(G)$ for which there exists no $F_{0} \in A^{p}(\Omega)$, such that $\left.F_{0}\right|_{G}=f_{0}$. Then the set $A(p, G)$ of functions $f \in A^{p}(G)$ for which there exists no $F \in A^{p}(\Omega)$ such that $\left.F\right|_{G}=f$ is an open and dense subset of $A^{p}(G)$.

Proof First, we will prove that the set $S=A^{p}(G) \backslash A(p, G)$ is closed. Let $\left(h_{n}\right)_{n \geq 1}$ be a sequence in $S$ converging in the topology of $A^{p}(G)$ to a function $h \in A^{p}(G)$. By the maximum modulus principle, the extensions $H_{n}$ of $h_{n}$ form a uniformly Cauchy sequence on $\bar{\Omega}$. Thus, the limit $H$ of $H_{n}$ on $\bar{\Omega}$ is an extension of $h$. Therefore $g \in S$ and $S$ is a closed subset of $A^{p}(G)$.

Now we will prove that the set $S$ has empty interior. If $S$ does not have empty interior, then there is a function $f$ in the interior of $S$ and $l \in\{0,1, \ldots\}, l \leq p$ and $d>0$, such that

$$
\left\{f \in A^{p}(G): \sup _{z \in \bar{G}}\left|f^{(j)}(z)-g^{(j)}(z)\right|<d, 0 \leq j \leq l \mid\right\} \subset S .
$$

Obviously, $f_{0}$ is not identically equal to zero which implies

$$
m=\max \left\{\sup _{z \in \bar{G}}\left|f_{0}^{(j)}(z)\right|, j=0,1, \ldots, l\right\}>0 .
$$

From the definitions, the function $h(z)=f(z)+\frac{d}{2 m} f_{0}(z), z \in \bar{G}$ belongs to $S$. Since $f, h$ belong to $S$, there are $F, H \in A^{p}(\Omega)$, such that $\left.F\right|_{G}=f,\left.H\right|_{G}=h$. Then $\frac{2 m}{d}(H(z)-F(z))$ belongs to $A^{p}(\Omega)$ and is equal to $f_{0}$ in $G$ which contradicts our hypothesis. Thus the set $S$ has empty interior. 
Remark 7.2 If the interior of $L$ in $\mathbb{C}$ is non-empty, then there always exists a function $f_{0} \in A^{p}(G)$ for which there does not exist a function $F_{0} \in A^{p}(\Omega)$, such that $\left.F_{0}\right|_{G}=$ $\left.f_{0}\right|_{G}$.

Remark 7.3 From the previous results, we have a dichotomy: Either every $f \in A^{p}(G)$ has an extension in $A^{p}(\Omega)$ or generically all functions $f \in A^{p}(G)$ do not admit any extension in $A^{p}(\Omega)$. The first case holds if $a_{p}(L)=0$ and the second case if $a_{p}(L)>0$ (Theorem 3.12).

Remark 7.4 In a similar way, we can prove that if $L$ is a compact set contained in the open set $U$, then either every $f \in \tilde{A}^{p}(U \backslash L)$ has an extension in $\tilde{A}^{p}(U)$ or generically every $f \in \tilde{A}^{p}(U \backslash L)$ does not have an extension in $\tilde{A}^{p}(U), p \in\{0,1,2, \ldots\} \cup\{\infty\}$. The first horn of this dichotomy holds if and only if $\tilde{a}_{p}(L)=0$ which is equivalent with the fact that the interior of $L$ is void in $\mathbb{C}$ (Theorem 3.16).

Now we present some local versions of the above results.

Definition 7.5 Let $L$ be a compact subset of $\mathbb{C}$ and $U$ be an open subset of $\mathbb{C}$, such that $L \subseteq U$. Let also $z_{0} \in \partial L$. A function $f \in H(U \backslash L)$ is extendable at $z_{0}$ if there exists $r>0$ and $F \in H\left(D\left(z_{0}, r\right)\right)$ such that $\left.F\right|_{(U \backslash L) \cap D\left(z_{0}, r\right)}=\left.f\right|_{(U \backslash L) \cap D\left(z_{0}, r\right)}$. Otherwise, $f$ is not extendable at $z_{0}$.

Below we will use the above definition of extendability.

Proposition 7.6 Let $L$ be a compact subset of $\mathbb{C}$ and $U$ be an open subset of $\mathbb{C}$, such that $L \subseteq U$. Let also $M$ and $r$ be positive real numbers, $p \in\{0,1,2, \ldots\} \cup\{\infty\}$ and $z_{0} \in \partial L$. The set $E_{M, p, U, L, z_{0}, r}$ of functions $f \in A^{p}(U \backslash L)$ such that there is $F \in H\left(D\left(z_{0}, r\right)\right)$ with $\left.F\right|_{(U \backslash L) \cap D\left(z_{0}, r\right)}=\left.f\right|_{(U \backslash L) \cap D\left(z_{0}, r\right)}$ and $\|F\|_{\infty} \leq$ M is a closed subset of $A^{p}(U \backslash L)$. Also, if there exists $f_{0} \in A^{p}(U \backslash L)$ which is not extendable at $z_{0}$, then the interior of $E_{M, p, U, L, z_{0}, r}$ is void in $A^{p}(U \backslash L)$.

Proof We will first prove that the set $E_{M, p, U, L, z_{0}, r}$ is a closed subset of $A^{p}(U \backslash L)$. Let $\left(f_{n}\right)_{n \geq 1}$ be a sequence in $E_{M, p, U, L, z_{0}, r}$ converging in the topology of $A^{p}(U \backslash L)$ to a function $f \in A^{p}(U \backslash L)$. Without of loss of generality we assume that $U$ is bounded. This implies that $f_{n}$ converges uniformly on $\overline{U \backslash L}$ to $f$ and that there exists a sequence $\left(F_{n}\right)_{n \geq 1}$ in $H\left(D\left(z_{0}, r\right)\right)$ such that $\left.F_{n}\right|_{(U \backslash L) \cap D\left(z_{0}, r\right)}=\left.f_{n}\right|_{(U \backslash L) \cap D\left(z_{0}, r\right)}$ and $\|F\|_{\infty} \leq M$ for every $n \geq 1$. By Montel's Theorem there exists a subsequence of $\left(F_{n}\right),\left(F_{k_{n}}\right)$, which converges uniformly on the compact subsets of $D\left(z_{0}, r\right)$ to a function $F$ which is holomorphic and bounded by $M$ on $D\left(z_{0}, r\right)$. Since $F_{k_{n}}$ converges to $f$ on $(U \backslash L) \cap D\left(z_{0}, r\right)$, the functions $f$ and $F$ are equal on $(U \backslash L) \cap D\left(z_{0}, r\right)$. Thus $f$ belongs to $E_{M, p, U, L, z_{0}, r}$ and $E_{M, p, U, L, z_{0}, r}$ is a closed subset of $A^{p}(U \backslash L)$. If there exists $f_{0} \in A^{p}(U \backslash L)$ which is not extendable at $z_{0}$, the interior of $E_{M, p, U, L, z_{0}, r}$ is void in $A^{p}(U \backslash L)$, the proof of which is similar to the proof of Theorem 7.1.

Here we have another dichotomy which is a local version of the first one.

Theorem 7.7 Let $L$ be a compact subset of $\mathbb{C}$ and $U$ be an open subset of $\mathbb{C}$, such that $L \subseteq U$ and let $p \in\{0,1,2, \ldots\} \cup\{\infty\}, z_{0} \in \partial L$. The set $E_{p, U, L, z_{0}}=$ $\bigcup_{M=1}^{\infty} \bigcup_{n=1}^{\infty} E_{M, p, U, L, z_{0}, \frac{1}{n}}$ is the set of extendable functions of $A^{p}(U \backslash L)$ at $z_{0}$. Then 
(i) either every function $f \in A^{p}(U \backslash L)$ is extendable at $z_{0}$

(ii) or generically all functions $f \in A^{p}(U \backslash L)$ are not extendable at $z_{0}$.

Proof If $(i)$ is not true, then Proposition 7.6 shows that $E_{M, p, U, L, z_{0}, \frac{1}{n}}$ is closed with empty interior for all natural numbers $n \geq 1, M \geq 1$. Then

$$
A^{p}(U \backslash L) \backslash E_{p, U, L, z_{0}}=\bigcap_{M=1}^{\infty} \bigcap_{n=1}^{\infty}\left(A^{p}(U \backslash L) \backslash E_{M, p, U, L, z_{0}, \frac{1}{n}}\right)
$$

is the intersection of a countable number of open and dense subsets of $A^{p}(U \backslash L)$ and Baire's Theorem shows that $A^{p}(U \backslash L) \backslash E_{p, U, L, z_{0}}$, which coincides with the set of non-extendable functions of $A^{p}(U \backslash L)$ at $z_{0}$, is a dense and $G_{\delta}$ subset of $A^{p}(U \backslash L)$.

In what follows, we compare two notions: local extendability and existence of a holomorphic extension. At first, we examine the case of a compact set $L$ with empty interior.

Proposition 7.8 Let $L$ be a compact subset of $\mathbb{C}$ and $U$ be an open subset of $\mathbb{C}$, such that $L \subseteq U$ and $L^{\circ}=\emptyset$. Let also $f \in H(U \backslash L)$. Then $f$ is extendable at every $z_{0} \in \partial L$ if and only if there exists a holomorphic extension $F$ of $f$ on $U$. If additionally $f \in A^{p}(U \backslash L)$ for some $p \in\{0,1, \ldots\} \cup\{\infty\}$, then $F \in A^{p}(U)$.

Proof If there exists a holomorphic extension $F$ of $f$ on $U$, then obviously $f$ is extendable at every $z_{0} \in \partial L=L$.

Conversely, if $f$ is extendable at every $z_{0} \in L$, then for every $z_{0} \in L$ there is a holomorphic function $F_{z_{0}}$ defined on $D\left(z_{0}, r_{z_{0}}\right) \subseteq U$ such that $\left.F_{z_{0}}\right|_{(U \backslash L) \cap D\left(z_{0}, r_{z_{0}}\right)}=$

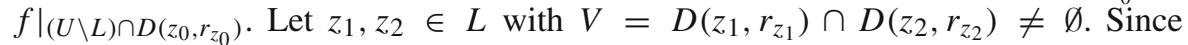
$L^{\circ}=\emptyset, V \backslash L$ is a non-empty open set, $F_{z_{1}}, F_{z_{2}}$ are holomorphic on the domain $V$ and coincide with $f$ on $V \backslash L$. By analytic continuation, $F_{z_{1}}=F_{z_{2}}$ on $V$. So, the function $F$ defined on $U$ such that $F(z)=F_{z}(z)$ for every $z \in L$ and $F(z)=f(z)$ for every $z \in U \backslash L$ is a holomorphic extension of $f$ on $U$. Obviously, if $f \in$ $A^{p}(U \backslash L)$, then $F \in A^{p}(U)$.

Remark 7.9 Whenever $L^{\circ} \neq \emptyset$, the equivalence of Proposition 7.8 is not true. Indeed if $w \in L^{\circ} \neq \emptyset$, then the holomorphic function $f(z)=\frac{1}{z-w}$ for $z \in U \backslash L$ cannot be extended to a holomorphic function on $U$, but it is extendable at every $z_{0} \in \partial L$.

We consider again a compact set $L \subseteq \mathbb{C}$ and an open set $U \subseteq \mathbb{C}$, such that $L \subseteq U$ and a $p \in\{0,1,2, \ldots\} \cup\{\infty\}$. Now we want to find a similar connection between $a_{p}(L)$ and $a_{p}\left(L \cap \overline{D\left(z_{0}, r\right)}\right)$; that is, is the condition $a_{p}(L)=0$ equivalent to the condition $a_{p}\left(L \cap \overline{D\left(z_{0}, r\right)}\right)=0$ for all $z_{0} \in L$ ?

If we suppose that $L^{\circ} \neq \emptyset$, then there exist $z_{0}$ and $r>0$ such that $D\left(z_{0}, r\right) \subseteq L$. Thus $a_{p}(L)$ and $a_{p}\left(L \cap \overline{D\left(z_{0}, r\right)}\right)$ are strictly positive.

So, we need not assume that $L^{\circ}=\varnothing$, since it follows from both the conditions $a_{p}(L)=0$ and $a_{p}\left(L \cap \overline{D\left(z_{0}, r\right)}\right)=0$ for every $z_{0} \in L$ and for some $r=r_{z_{0}}>0$. Also, the first condition obviously implies the second one. 
Probably Theorem 3.6 holds even for $p \geq 1$. Specifically, if $a_{p}(L)=0$ and $V$ is an open set, then every function $g \in A^{p}(V \backslash L)$ belongs to $A^{p}(V)$. This leads us to believe that the above conditions are in fact equivalent. However, this will be examined in future papers.

Acknowledgements We would like to thank A. Borichev, P. Gauthier, J.P. Kahane, V. Mastrantonis, P. Papasoglu, and A. Siskakis for helpful communications.

Open Access This article is distributed under the terms of the Creative Commons Attribution 4.0 International License (http://creativecommons.org/licenses/by/4.0/), which permits unrestricted use, distribution, and reproduction in any medium, provided you give appropriate credit to the original author(s) and the source, provide a link to the Creative Commons license, and indicate if changes were made.

\section{References}

1. Bolkas, E., Nestoridis, V., Panagiotis, C., Papadimitrakis, M.: One sided extendability and $p$-continuous analytic capacities. arXiv:1606.05443v2

2. Bolkas, E., Nestoridis, V., Panagiotis, C.: Non extendability from any side of the domain of definition as a generic property of smooth or simply continuous functions on an analytic curve. arXiv:1511.08584

3. Cater, F.S.: Differentiable, nowhere analytic functions. Am. Math. Mon. 91(10), 618-624 (1984)

4. Daghighi, A., Krantz, S.: A note on a conjecture concerning boundary uniqueness (2015). arXiv:1407.1763v2 [math.CV]

5. Gamelin, T.: Uniform Algebras. AMS Chelsea Publishing, Providence, RI (2005)

6. Garnett, J.: Positive length but zero analytic capacity. Proc. Am. Math. Soc. 24, 696-699 (1970)

7. Garnett, J.: Analytic Capacity and Measure. Lecture Notes in Mathematics, vol. 277. Springer, Berlin (1972)

8. Krantz, S., Parks, H.: A Primer of Real Analytic Functions, 2nd edn. Birkhauser, Boston (2002)

9. Nestoridis, V.: Non extendable holomorphic functions. Math. Proc. Camb. Philos. Soc. 139(2), 351-360 (2005)

10. Nestoridis, V., Papadopoulos, A.: Arc length as a global conformal parameter for analytic curves. JMAA 445(2), 1505-1515 (2017)

11. Newmann, M.H.A., M. A., F. R.S.: Elements of the Topology of Plane Sets of Points. Cambridge University Press, Cambridge (1954)

12. Rudin, W.: Function Theory in Polydiscs. W. A. Benjamin, New York (1969)

13. Whitney, H.: Analytic extensions of differentiable functions defined in closed sets. Trans. Am. Math. Soc. 36, 63-89 (1934)

14. Zalcman, L.: Analytic Capacity and Rational Approximation. Lecture Notes in Math., vol. 50, Springer, Berlin (1968) 